\title{
EQUATIONAL COMPACTNESS OF BI-FRAMES AND PROJECTION ALGEBRAS
}

\author{
Friedrich WEHRUNG \\ Université de Caen \\ Département de Mathématiques \\ 14032 CAEN CEDEX \\ FRANCE
}

\begin{abstract}
We generalize D. Kelly's and K.A. Nauryzbaev's results of 1-variable and 2-variable equational compactness of complete distributive lattices satisfying the infinite distributive law and its dual ("bi-frames") to objects similar to monadic algebras (which we will call projection algebras). This will lead us in particular to an example of bi-frame that is not 3-variable equationally compact, even for countable equation systems, thus solving a problem of G. Grätzer. This example is realized as a certain complete sublattice of the complete Boolean algebra of regular open subsets of some Polish space.
\end{abstract}

\section{$\S$ O. INTRODUCTION.}

D. Kelly showed in 1972 that every complete distributive lattice that is meet- and join-continuous (from now on a bi-frame, to adopt the terminology of [16]) is 1-variable equationally compact; the problem whether one could replace 1-variable equational compactness by full equational compactness was left open there (in the form of a question mark in the recapitulative list of the known results at that time). It is probably in G. Grätzer's book [11] that was first stated explicitely the problem of extending Kelly's result. Actually, the problem was not stated "determine whether...", but "prove that" "a distributive lattice is equationally compact if and only if it is complete and satisfies both the infinite distributive law and its dual" [11, p. 128], and indeed, a positive answer seemed much more likely. In 1986, K.A. Nauryzbaev partially vindicated Grätzer's conjecture by extending Kelly's result to 2 variables [19]. However, we find that it is not true for 3 variables. Our example (theorem 4.19 below) is the space of regular open lower sets of a certain preordered Polish space; it is not even 3-variable countably equationally compact. Although this example is not a Stone lattice (as defined in [11]), adjoining an infinitesimal element yields easily a bi-frame that is a Stone lattice but not 3-variable equationally compact (corollary 4.20).

We recall now some basic definitions and known results about equational compactness in ordered structures.

Let $\mathfrak{M}$ be a model for a first-order language $\mathcal{L}$. If $\mathfrak{m}$ is a cardinal number, we say that $\mathfrak{M}$ is $\mathfrak{m}$-variable atomic compact (resp. $\mathfrak{m}$-variable countably atomic compact) when for every (resp. countable) system $\Gamma$ of atomic formulæ with parameters from $\mathfrak{M}$ and

1991 Mathematics subject classification: primary 08A45, 03G15, 06D20, 06B35, 06B30, 06D10, 28A05, 03C20; secondary 03F55.

Key words and phrases: equational compactness, monadic algebras, bi-frames, partially ordered sets, lower sets, Polish spaces, regular open sets, Baire property. 
variables ("unknowns") from a given set of cardinal (at most) $\mathfrak{m}$, if every finite subset of $\Gamma$ admits a solution in $\mathfrak{M}(\mathfrak{M}$ is finitely solvable), then $\Gamma$ admits a solution in $\mathfrak{M}$. We refer to $[\mathbf{1}, \mathbf{2 3}, \mathbf{2 4}]$ for the general theory of atomic compactness. In particular, a model is atomic compact if and only if it is a retract of all its reduced powers (resp. ultrapowers). When there are no relation symbols (or when the relations are definable from the operations with atomic formulæ), we will follow the current terminology by saying equational compactness instead of atomic compactness.

Roughly speaking, atomic compactness is the amount of algebraic information that could be inferred from topological compactness if the latter were present (which is not always the case). It is well-known that a topologically compact model (i.e. such that the interpretations of the relation symbols are closed and the interpretations of the operation symbols are continuous for some compact Hausdorff topology on the model) is always atomic compact; so are the retracts of topologically compact models. But the converse does not hold. We refer to [13] for information and examples about this (among other things!).

In the case of ordered structures, perhaps that the most well-known result of equational compactness is Sikorski's extension theorem [22], which states that a Boolean algebra is complete if and only if it is injective (thus if and only if it is equationally compact). In case of semilattices, there exists also a complete answer [12]. See also [4] for the case of semilattices with distinguished endomorphisms, or [25] where atomic compactness of lattices is considered in the language $(\leq)$ (without $\wedge$ and $\vee$ ). Positive results on equational compactness properties of ordered groups and related structures have been obtained (sometimes with the help of forcing) in $[\mathbf{2 6}, \mathbf{2 7}, \mathbf{2 8}]$.

At that point comes a remarkable fact: most of natural ordered algebraic structures arising from Boolean algebras are not topologically compact (and not even locally compact); there are noticeable exceptions: for example many weak topologies on (ordered) Banach spaces (but the unit spheres are not closed so that they cannot be used directly for $\mathbb{N}$-valued functions, see the example further in the Introduction); or the Hausdorff topology on the set of closed subsets of a compact Hausdorff topological space (but the intersection map is not continuous in general); or completely distributive lattices [16, VII 1.10] (but these are fairly special objects). Perhaps the best reason for this is the result of P.S. Rema [21] saying that the only topologically compact Boolean algebras are the complete atomic ones (i.e. the $\mathcal{P}(\Omega)$ for any set $\Omega$ ). See also $[\mathbf{9}]$ to show that order topologies are in general not suitable for a topological study. This could be laconically summarized by saying that for ordered algebraic structures, topological compactness is exceptional.

On the contrary, the aforementioned $[4, \mathbf{1 2}, \mathbf{2 2}, \mathbf{2 6}, \mathbf{2 7}, \mathbf{2 8}]$ (among others) show that ordered algebraic structures enjoying some property of completeness also enjoy a corresponding property of atomic compactness. This would prompt us (a little hastily) to claim that in complete ordered algebraic structures, atomic compactness is the general rule. The surprise coming up is that there are as we shall see counterexamples to this claim, without any pathological character.

As central structures for the study of ordered algebraic structures, lattices play a special role, a possible explanation for this being the fact that the collection of all congruences on a given algebra is a lattice. Say that a complete lattice $A$ is meet-continuous 
(resp. join-continuous) when for every $a \in A$ and every subset $X$ of $A$ that is directed upward (resp. downward), we have $a \wedge \bigvee X=\bigvee(a \wedge X)$ (resp. $a \vee \wedge X=\bigwedge(a \vee X))$. It is proved in $[\mathbf{1 2}]$ that every 1-variable equationally compact lattice is both meet-continuous and join-continuous (contrarily to $[\mathbf{1 7}, \mathbf{1 9}]$, we will not say "continuous" in order to avoid confusion with the notion of continuous lattice studied for example in $[\mathbf{1 0}, \mathbf{1 6}])$. The converse is false even for modular lattices [17]. On the other hand, D. Kelly proved in [17] that every complete distributive lattice which is both meet-continuous and join-continuous is 1-variable equationally compact, leaving open the problem of full equational compactness. The problem was restated more explicitely in G. Grätzer's book [11, problem II.23, page 128] ("prove that a distributive lattice is equationally compact if and only if it is complete and satisfies the infinite distributive law and its dual"). This bears also an intriguing connection with the fact that if $H$ is a complete Heyting algebra, then names of ultrafilters on sets in $\mathbf{V}$ in the Heyting-valued universe $\mathbf{V}^{H}$ correspond precisely to the retractions of reduced powers of $H$ : if $I$ is a set in $\mathbf{V}, \mathcal{F}$ is a filter on $I$ in $\mathbf{V}$ and $\mathcal{U}$ is a $H$-valued name such that $\| \mathcal{U}$ is a ultrafilter on $\check{I}$ and $\check{\mathcal{F}} \subseteq \mathcal{U} \|=1$ (one has to be careful about the definition of a ultrafilter in $\mathbf{V}^{H}$ since the latter is no longer a model of classical logic), then one can define a retraction of the reduced power ${ }^{I} H / \mathcal{F}$ onto $H$ by sending $\left[x_{i}: i \in I\right]_{\mathcal{F}}$ on $\|\dot{x} \in \mathcal{U}\|$ where $\dot{x}=\{(\check{\imath}, x(i)): i \in I\}$. Conversely, if $\mu$ is a map from ${ }^{I} H$ to $H$ invariant under $\mathcal{F}$-equivalence inducing a retraction from ${ }^{I} H / \mathcal{F}$ to $H$, then $\mathcal{U}=\left\{(\dot{x}, \mu(x)): x \in{ }^{I} H\right\}$ is the $H$-valued name of a ultrafilter on $I$. Thus equational compactness of $H$ is very closely related to the satisfaction in the $H$-valued universe $\mathbf{V}^{H}$ of the ultrafilter theorem. This remark has been used in the case of Boolean algebras in $[\mathbf{2 6}, \mathbf{2 7}]$; see also $[\mathbf{2}]$.

A significant progress has been made towards a positive solution to Grätzer's problem by K.A. Nauryzbaev in [19], who proved that every complete distributive lattice which is meet-continuous and join-continuous is 2-variable equationally compact. We shall follow the current terminology by calling bi-frames those complete distributive lattices which are both meet-continuous and join-continuous [16, page 284]. The remarkable point about Kelly's and Nauryzbaev's solutions is that they are both constructive, i.e. a global solution of the atomic system under consideration is defined (via the lattice operations and $\overline{\lim }$ and $\underline{\mathrm{lim}}$ ) from a family of solutions of partial subsystems. Elaborating on this remarkable fact, we shall present in section 1 an axiomatic context ("projection algebras"), reminiscent of monadic algebras [15], but generalizing them and also the case of the embedding of a bi-frame into a reduced power. In this more general context, Kelly's and Nauryzbaev's solutions will be formulated as mere first-order statements; in particular, they will yield consequences on arbitrary (and not necessarily complete) structures, see corollary 1.8. To conclude section 1, we will clarify the concept of ultracontinuous lattice introduced in [19] to provide a sufficient condition for equational compactness. In fact, ultracontinuity will yield equational characterizations of those lattices with compact Hausdorff interval topology (proposition 1.10) or of those lattices that are made into compact Hausdorff topological lattices with their interval topology (proposition 1.13).

In this paper, we will also settle Grätzer's problem by finding an example of bi-frame that is not 3-variable equationally compact (and not even 3-variable countably equationally compact). This bi-frame will be realized as the algebra of those regular open subsets of 
some preordered Polish space $\Omega$ that are lower subsets of $\Omega$ (theorem 4.19). This result will allow us to conclude immediately (with the same conclusion) for the class of bi-frames that are in addition Stone lattices (corollary 4.20).

In order to give an idea of how this example works, we give here a much simpler example (in another language). Equip $\Omega=\mathcal{P}(\omega)$ with its natural topology (homeomorphic to the Cantor space) and let $\lambda$ be the Lebesgue probability measure on $\Omega$. Let $B$ be the complete Boolean algebra of $\lambda$-measurable subsets of $\Omega$ modulo $\lambda$-null sets. Furthermore, equip $\Omega$ with its natural structure of Boolean ring (with $x \cdot y=x \cap y$ and $x+y=$ $(x \cap \complement y) \cup(y \cap \complement x))$. Let $A=\{a \subseteq \omega: a$ is finite $\}$; for all $a \in A \cup\{\omega\}$, let $\tau_{a}$ be the automorphism of $B$ induced by the translation of $\Omega$ defined by $x \mapsto a+x$. Then the following holds (compare with $[4]$ ):

The (complete) Boolean algebra $B$ together with the automorphisms $\tau_{a}(a \in A \cup\{\omega\})$ is not 1-variable countably equationally compact.

The proof of this fact is very simple and runs as follows. Consider the following equation system with one unknown $\mathbf{x}$ :

$$
\left\{\begin{array}{l}
\mathbf{x} \wedge \tau_{\omega} \mathbf{x}=0 ; \\
\mathbf{x} \vee \tau_{\omega} \mathbf{x}=1 ; \\
\tau_{a} \mathbf{x}=\mathbf{x} \quad(\text { all } a \text { in } A)
\end{array}\right.
$$

Then this system is finitely solvable (consider for every $n \in \omega$ the element of $B$ corresponding to $U_{n}=\{x \in \Omega: n \in x\}$ ). However, it is not solvable, otherwise there would exist a measurable subset $X$ of $\Omega$ such that $X$ is a tail set [20, page 84] and $X \cap \tau_{\omega} X$ and $\Omega \backslash\left(X \cup \tau_{\omega} X\right)$ are null, but this would contradict the zero-one law. This example shows also where weak topology breaks down (the sequence of characteristic functions of the $U_{n}$ 's weakly converges to $1 / 2$ ). A similar argument shows that the analogue of this example for category is also valid.

We shall use standard notations and terminology. If $X$ and $Y$ are sets, then we will denote by $X \triangle Y=(X \backslash Y) \cup(Y \backslash X)$ their symmetrical difference, and by ${ }^{X} Y$ the set of all maps from $X$ to $Y$. If $f$ is a map from $X \times Y$ to $Z$ and $(x, y) \in X \times Y$, then we will write $f(x, y)$ instead of $f((x, y))$, and similarly for several variables. Families will be denoted indifferently in the form $\left(x_{i}\right)_{i \in I}$ or $\left\langle x_{i}: i \in I\right\rangle$. We will denote by $\omega$ the set of all natural numbers, and natural numbers may be identified with the set of their strict predecessors.

If $P$ is a set endowed with a binary relation $\leq$ and $X \subseteq P$, then we write $\downarrow X=\{y \in$ $P:(\exists x \in X)(y \leq x)\}$ and $\uparrow X=\{y \in P:(\exists x \in X)(y \geq x)\} ; X$ is a lower subset (resp. upper subset) of $P$ when $X=\downarrow X$ (resp. $X=\uparrow X)$. Furthermore, $P$ is connected when the only subsets of $P$ that are both lower and upper sets are $\emptyset$ and $P$.

Following the convention in G. Birkhoff's book [3], our lattices will not be assumed to have a least or a largest element (although it will most of the time be the case). If $\kappa$ is a cardinal, then we will say that a $\kappa$-bi-frame is a $\kappa$-complete distributive lattice satisfying both conditions of meet-continuity and join-continuity for subsets $X$ of cardinal $<\kappa ; \omega_{1}$-bi-frames will be called $\sigma$-bi-frames. Thus this terminology is consistent with the terminology concerning Boolean algebras. 
If $\Omega$ is a topological space and $X$ is a subset of $\Omega$, we will denote by $\operatorname{Cl}(X)$ (resp. $\operatorname{Int}(X))$ the topological closure (resp. interior) of $X$. We will say that $X$ is clopen when it is both open and closed, regular open when $X=\operatorname{Int} \mathrm{Cl}(X)$; that $X$ has the Baire property when there exists an open set $U$ such that $U \triangle X$ is meager [20]. If $\varphi(\mathbf{x})$ is a formula (with parameters) with one free variable $\mathbf{x}$ and $\Omega$ is a topological space, then $\left(\forall^{*} x \in \Omega\right) \varphi(x)$ (resp. $\left.\left(\exists^{*} x \in \Omega\right) \varphi(x)\right)$ will be the statement " $\{x \in \Omega: \varphi(x)\}$ is residual" (resp. non meager). Note that if $\{x \in \Omega: \varphi(x)\}$ has the Baire property, then $\left(\exists^{*} x \in \Omega\right) \varphi(x)$ is equivalent to $\left(\forall^{*} x \in U\right) \varphi(x)$ for a certain non-meager open subset $U$ of $\Omega$. If $X$ and $Y$ are Polish spaces and $f$ is a map from $X$ to $Y, f$ has the Baire property when for every open subset $V$ of $Y, f^{-1}[V]$ has the Baire property in $X$.

Finally, we refer to [5] for the basic facts about reduced products, reduced powers, ultraproducts, etc.. In particular, if $\mathcal{F}$ is a filter on a set $I,\left(M_{i}\right)_{i \in I}$ is a $I$-family of sets and $\left(x_{i}\right)_{i \in I} \in \prod_{i \in I} M_{i}$, then we will denote by $\left[x_{i}: i \in I\right]_{\mathcal{F}}$ the corresponding element of the reduced product $\prod_{\mathcal{F}}\left(M_{i}\right)_{i \in I}$.

\section{§1. KELLY AND NAURYZBAEV. PROJECTION ALGEBRAS.}

We start with the following

1.1. Definition. A projection algebra is a structure $\left(A, \wedge, \vee,{ }^{+},{ }^{-}\right)$satisfying the following properties.

(PA0) $(A, \wedge, \vee)$ is a distributive lattice, and ${ }^{+}$and ${ }^{-}$are unary operations on $A$.

As usual, define the natural ordering of $A$ by $x \leq y \Leftrightarrow x \wedge y=x \Leftrightarrow x \vee y=y$.

$(\operatorname{PA} 1 \wedge)(\forall \mathbf{x}, \mathbf{y})\left((\mathbf{x} \vee \mathbf{y})^{+}=\mathbf{x}^{+} \vee \mathbf{y}^{+}\right)$

$(\mathrm{PA} 1 \vee)(\forall \mathbf{x}, \mathbf{y})\left((\mathbf{x} \wedge \mathbf{y})^{-}=\mathbf{x}^{-} \wedge \mathbf{y}^{-}\right)$

$(\mathrm{PA} 2 \wedge)(\forall \mathbf{x}, \mathbf{y})\left(\mathbf{x}^{+} \wedge \mathbf{y}^{-} \leq(\mathbf{x} \wedge \mathbf{y})^{+}\right) ;$

$(\mathrm{PA} 2 \vee)(\forall \mathbf{x}, \mathbf{y})\left((\mathbf{x} \vee \mathbf{y})^{-} \leq \mathbf{x}^{+} \vee \mathbf{y}^{-}\right)$

$(\mathrm{PA} 3+)(\forall \mathbf{x})\left(\mathbf{x}^{++}=\mathbf{x}^{+-}=\mathbf{x}^{+}\right)$;

(PA3-) $(\forall \mathbf{x})\left(\mathbf{x}^{--}=\mathbf{x}^{-+}=\mathbf{x}^{-}\right)$.

This axiom system bears close similarities with the axiom system defining monadic algebras [15]: this similarity would suggest to write $\exists x$ instead of $x^{+}$and $\forall x$ instead of $x^{-}$. There are two reasons why we shall not do so. The first one is for sake of clarity of notation; the second one is that projection algebras do not necessarily satisfy $(\forall \mathbf{x})\left(\mathbf{x} \leq \mathbf{x}^{+}\right)$ (or $(\forall \mathbf{x})\left(\mathbf{x}^{-} \leq \mathbf{x}\right)$ ), which would be rather counter-intuitive for quantifiers.

Note also that projection algebras satisfy as for lattices a duality principle: namely, if $\left(A, \wedge, \vee,{ }^{+},{ }^{-}\right)$is a projection algebra, then $\left(A, \vee, \wedge,{ }^{-},{ }^{+}\right)$is a projection algebra. Thus, the dual of a theorem of projection algebras is a theorem of projection algebras.

1.2. Lemma. Every projection algebra satisfies the following statements:

(a) $(\forall \mathbf{x}, \mathbf{y})\left(\mathbf{x} \leq \mathbf{y} \Rightarrow\left(\mathbf{x}^{+} \leq \mathbf{y}^{+}\right.\right.$and $\left.\left.\mathbf{x}^{-} \leq \mathbf{y}^{-}\right)\right)$;

(b) $(\forall \mathbf{x})\left(\mathbf{x}^{-} \leq \mathbf{x}^{+}\right)$;

(c) The following statements with one free variable $\mathbf{x}$ are equivalent: 
(i) $\mathbf{x}^{+}=\mathbf{x}$;

(i) $\mathbf{x}^{-}=\mathbf{x}$;

(iii) $(\exists \mathbf{y})\left(\mathbf{x}=\mathbf{y}^{+}\right)$;

(iv) $(\exists \mathbf{y})\left(\mathbf{x}=\mathbf{y}^{-}\right)$.

Proof. (a) follows immediately from $(\mathrm{PA} 1 \wedge)$ and (PA1 $\mathrm{V})$.

(b) For all $x$ in a given projection algebra, we have $x^{-} \leq\left(x \vee x^{+}\right)^{-}$by (a). By (PA2 $\vee$ ), we have $\left(x \vee x^{+}\right)^{-} \leq x^{+} \vee x^{+-}$; since $x^{+-}=x^{+}$by $(\mathrm{PA} 3+)$, we obtain $\left(x \vee x^{+}\right)^{-} \leq x^{+}$, and the result follows.

(c) An easy consequence of (PA3+) and (PA3-).

An element $x$ of a projection algebra is central when $x^{+}=x$ (or, equivalently by lemma 1.2, when $x^{-}=x$ ). Throughout this paper, we shall denote the set of all central elements of $A$ by $Z(A)$, and call it the center of $A$. There should be no confusion between this definition of center and the notion of center of a partially ordered set presented in $[\mathbf{3}]$ or the definition of centre of a lattice presented in [11, page 156].

1.3. Lemma. Let $A$ be a projection algebra. Then $Z(A)$ is a sublattice of $A$.

Proof. For all elements $x$ and $y$ of $A$, we have $x \wedge y=x^{-} \wedge y^{-}=(x \wedge y)^{-} \in Z(A)$, and $x \vee y=x^{+} \vee y^{+}=(x \vee y)^{+} \in Z(A)$.

1.4. Lemma. Let $A$ be a projection algebra. Then for all $x \in A$ and all $a \in Z(A)$, we have

$$
\begin{array}{ll}
(x \wedge a)^{+}=x^{+} \wedge a ; & (x \vee a)^{+}=x^{+} \vee a \\
(x \wedge a)^{-}=x^{-} \wedge a ; & (x \vee a)^{-}=x^{-} \vee a
\end{array}
$$

Proof. We have $(x \wedge a)^{+} \leq x^{+} \wedge a$ by lemma 1.2; conversely, we have $x^{+} \wedge a=x^{+} \wedge a^{-} \leq$ $(x \wedge a)^{+}$by $(\mathrm{PA} 2 \wedge)$. Thus $(x \wedge a)^{+}=x^{+} \wedge a$. Similarly, $a \vee x^{-} \leq(a \vee x)^{-}$by lemma 1.2, and conversely, using (PA2 $\vee$ ), we obtain $(a \vee x)^{-} \leq a^{+} \vee x^{-}=a \vee x^{-}$. Both remaining identities result immediately from $(\mathrm{PA} 1 \wedge)$ and $(\mathrm{PA} 1 \vee)$.

\subsection{Examples.}

(1) Let $(B, \exists)$ be a monadic algebra $[\mathbf{1 5}]$, i.e. a Boolean algebra $B$ endowed with a unary operation $\exists$ on $B$ such that for all $x, y$ in $B$, the following holds:

$$
\exists 0=0 ; \quad x \leq \exists x ; \quad \exists x=\exists \exists x ; \quad \exists(x \vee y)=\exists x \vee \exists y ; \quad \exists(x \vee \exists y)=\exists x \vee \exists y .
$$

For all $x$ in $B$, put $x^{+}=\exists x$ and $x^{-}=\forall x=\neg \exists \neg x$. Then $\left(B, \wedge, \vee,^{+},{ }^{-}\right)$is a projection algebra. More particularly, let $E$ be an equivalence relation on a set $\Omega$. For every element $x$ of $\Omega$, denote by $[x]$ the $E$-equivalence class of $x$. For every $X \subseteq \Omega$, let $\exists X$ be the 
$E$-saturated hull of $X$, i.e. $\exists X=\{x \in \Omega:[x] \cap X \neq \emptyset\}$. Then $(\mathcal{P}(\Omega), \exists)$ is a monadic algebra. The corresponding projection algebra is defined by

$$
(\forall X \subseteq \Omega) \begin{cases}X^{+}=\{x \in \Omega: & [x] \cap X \neq \emptyset\}, \\ X^{-}=\{x \in \Omega:[x] \subseteq X\} .\end{cases}
$$

(2) Another related example of projection algebra is the following. Let a group $G$ act on a $|G|^{+}$-bi-frame $A$. For every $x \in A$, put $x^{+}=\bigvee_{g \in G} g x$ and $x^{-}=\bigwedge_{g \in G} g x$. Then $\left(A, \wedge, \vee,{ }^{+},{ }^{-}\right)$is a projection algebra. If in addition $A$ is a Boolean algebra, then $\left(A,{ }^{+}\right)$is a monadic algebra.

(3) Let $E$ be a bi-frame, and let $\mathcal{F}$ be a filter on a nonempty set $I$. Identify $E$ with its image by the natural embedding into its reduced power ${ }^{*} E={ }^{I} E / \mathcal{F}$. For every $x=\left[x_{i}: i \in I\right]_{\mathcal{F}}$ in ${ }^{*} E$, define respectively $x^{+}$and $x^{-}$by

$$
\left\{\begin{array}{l}
x^{+}=\varlimsup_{\mathcal{\operatorname { l i m } _ { F }}}\left(x_{i}\right)_{i}=\bigwedge_{X \in \mathcal{F}} \bigvee_{i \in X} x_{i}, \\
x^{-}=\underline{\lim }_{\mathcal{F}}\left(x_{i}\right)_{i}=\bigvee_{X \in \mathcal{F}} \bigwedge_{i \in X} x_{i}
\end{array}\right.
$$

We prove that $\left({ }^{*} E, \wedge, \vee,{ }^{+},-\right)$is a projection algebra, with center $E$. All axioms of projection algebras are trivial except perhaps $(\mathrm{PA} 2 \wedge)$ and $(\mathrm{PA} 2 \vee)$. Let $x, y$ in ${ }^{*} E$, with $y=\left[y_{i}: i \in I\right]_{\mathcal{F}}$. We have $y^{-}=\bigvee_{X \in \mathcal{F}} y_{X}$ where for every $X \in \mathcal{F}$, we put $y_{X}=\bigwedge_{i \in X} y_{i}$. For all $X$ in $\mathrm{F}$, we have

$$
\begin{aligned}
x^{+} \wedge y_{X} & =\left(x \wedge y_{X}\right)^{+} \quad \text { because } y_{X} \in E \\
& \leq(x \wedge y)^{+} \quad \text { because } y_{X} \leq y .
\end{aligned}
$$

Since this holds for every $X \in \mathcal{F}$, we obtain that $x^{+} \wedge y^{-} \leq(x \wedge y)^{+}$, thus $(\operatorname{PA} 2 \wedge)$. The proof for $(\mathrm{PA} 2 \vee)$ is similar. The fact that $E$ is the center of ${ }^{*} E$ is trivial.

Note that in this example, we have in addition that for all $x, x^{+}=\bigwedge_{E}\{y \in E: x \leq y\}$ and $x^{-}=\bigvee_{E}\{y \in E: y \leq x\}$, although very easy examples show that one may not have $x^{-} \leq x$ or $x \leq x^{+}$.

The next proposition can be considered as the projection algebra version of Kelly's result.

1.6. Proposition. Let $A$ be a projection algebra, let $\varphi(\mathbf{x})$ be an atomic formula with parameters from $Z(A)$. Then $A$ satisfies the statement

$$
(\forall \mathbf{x})\left[\varphi(\mathbf{x}) \Rightarrow\left(\varphi\left(\mathbf{x}^{+}\right) \text {and } \varphi\left(\mathbf{x}^{-}\right)\right)\right] .
$$

Proof. Since $A$ is a distributive lattice, it suffices to consider the case where $\varphi$ is of the form $a \wedge \mathbf{x} \leq b$ or $a \leq b \vee \mathbf{x}$ where $a$ and $b$ are elements of $Z(A)$. So let $x$ in $A$. If $a \wedge x \leq b$, then, using lemma 1.4, we obtain $a \wedge x^{+}=(a \wedge x)^{+} \leq b^{+}=b$, thus, since $x^{-} \leq x^{+}$, also $a \wedge x^{-} \leq b$. Similarly, if $a \leq b \vee x$, then $a=a^{-} \leq(b \vee x)^{-}=b \vee x^{-}$, thus, since $x^{-} \leq x^{+}$, also $a \leq b \vee x^{+}$. 
Taking $A$ to be a reduced power of a bi-frame (see example 1.5 (3) above), one obtains immediately Kelly's original result that every bi-frame is 1-variable equationally compact.

The next proposition can be considered as the projection algebra version of Nauryzbaev's result.

1.7. Proposition. Let $A$ be a projection algebra, let $\varphi(\mathbf{x}, \mathbf{y})$ be an atomic formula with parameters from $Z(A)$. Then $A$ satisfies the statement

$$
(\forall \mathbf{x}, \mathbf{y})\left[\varphi(\mathbf{x}, \mathbf{y}) \Rightarrow \varphi\left(\mathbf{x}^{+},(\mathbf{x} \wedge \mathbf{y})^{+} \vee \mathbf{y}^{-}\right)\right]
$$

Proof. Since $A$ is a distributive lattice, it suffices to consider the case where $\varphi(\mathbf{x}, \mathbf{y})$ is of the form $a \wedge \bigwedge_{i \in I} \mathbf{x}_{i} \leq b \vee \bigvee_{j \in J} \mathbf{x}_{j}$ where we put $\mathbf{x}_{0}=\mathbf{x}$ and $\mathbf{x}_{1}=\mathbf{y}$ and $a$ and $b$ are two elements of $Z(A), I$ and $J$ are two disjoint subsets of 2 (by convention, we put $a \wedge \bigwedge_{i \in \emptyset} \mathbf{x}_{i}=a$ and $\left.b \vee \bigvee_{j \in \emptyset} \mathbf{x}_{j}=b\right)$. Thus it suffices to consider the eight following cases:

Case 1. $\varphi(\mathbf{x}, \mathbf{y})$ is $a \wedge \mathbf{x} \leq b$.

If $a \wedge x \leq b$, then $a \wedge x^{+} \leq b$ by proposition 1.6 .

Case 2. $\varphi(\mathbf{x}, \mathbf{y})$ is $a \wedge \mathbf{y} \leq b$.

If $a \wedge y \leq b$, then $a \wedge\left((x \wedge y)^{+} \vee y^{-}\right) \leq a \wedge y^{+} \leq b$ by case 1.

Case 3. $\varphi(\mathbf{x}, \mathbf{y})$ is $a \leq b \vee \mathbf{x}$.

If $a \leq b \vee x$, then $a \leq b \vee x^{+}$by proposition 1.6.

Case 4. $\varphi(\mathbf{x}, \mathbf{y})$ is $a \leq b \vee \mathbf{y}$.

If $a \leq b \vee y$, then $a \leq b \vee y^{-}$by proposition 1.6, thus $a \leq b \vee\left((x \wedge y)^{+} \vee y^{-}\right)$.

Case 5. $\varphi(\mathbf{x}, \mathbf{y})$ is $a \wedge \mathbf{x} \leq b \vee \mathbf{y}$.

If $a \wedge x \leq b \vee y$, then $a \wedge x=x \wedge(a \wedge x) \leq x \wedge(b \vee y) \leq b \vee(x \wedge y)$, thus $a \wedge x^{+}=$ $(a \wedge x)^{+} \leq(b \vee(x \wedge y))^{+}=b \vee(x \wedge y)^{+} \leq b \vee\left((x \wedge y)^{+} \vee y^{-}\right)$.

Case 6. $\varphi(\mathbf{x}, \mathbf{y})$ is $a \wedge \mathbf{y} \leq b \vee \mathbf{x}$.

If $a \wedge y \leq b \vee x$, then $a \wedge y^{+} \leq b \vee(x \wedge y)^{+}$by the proof of previous case; thus $a \wedge\left((x \wedge y)^{+} \vee y^{-}\right) \leq a \wedge y^{+} \leq b \vee(x \wedge y)^{+} \leq b \vee x^{+}$.

Case 7. $\varphi(\mathbf{x}, \mathbf{y})$ is $a \wedge \mathbf{x} \wedge \mathbf{y} \leq b$.

If $a \wedge x \wedge y \leq b$, then $a \wedge(x \wedge y)^{+} \leq b$ by proposition 1.6. Furthermore, $x^{+} \wedge y^{-} \leq(x \wedge y)^{+}$ by $(\mathrm{PA} 2 \wedge)$. Therefore, we have $a \wedge x^{+} \wedge\left((x \wedge y)^{+} \vee y^{-}\right)=\left(a \wedge(x \wedge y)^{+}\right) \vee\left(a \wedge\left(x^{+} \wedge y^{-}\right)\right)=$ $a \wedge(x \wedge y)^{+} \leq b$.

Case 8. $\varphi(\mathbf{x}, \mathbf{y})$ is $a \leq b \vee \mathbf{x} \vee \mathbf{y}$.

If $a \leq b \vee x \vee y$, then $a \leq b \vee(x \vee y)^{-}$by proposition 1.6, thus $a \leq b \vee x^{+} \vee y^{-}=$ $b \vee x^{+} \vee\left((x \wedge y)^{+} \vee y^{-}\right)$.

Thus in every case, $\varphi(x, y)$ implies $\varphi\left(x^{+},(x \wedge y)^{+} \vee y^{-}\right)$. 
Note that by duality, $A$ also satisfies the statement

$$
(\forall \mathbf{x}, \mathbf{y})\left[\varphi(\mathbf{x}, \mathbf{y}) \Rightarrow \varphi\left(\mathbf{x}^{-},(\mathbf{x} \vee \mathbf{y})^{-} \wedge \mathbf{y}^{+}\right)\right]
$$

Taking $A$ to be a reduced power of a bi-frame (see example $1.5(3)$ ) above, one obtains immediately Nauryzbaev's result that every bi-frame is 2-variable equationally compact. But while equational compactness is a statement involving infinite objects and some completeness, Nauryzbaev's formula has consequences on finite (or in fact arbitrary) distributive lattices, as for example the following corollary shows. Note that a similar result has been used in [28, corollary 2.4] to prove an atomic compactness property of monotone $\sigma$-complete groups.

1.8. Corollary. Let $A$ be a distributive lattice, let $\left\langle\varphi_{i}(\mathbf{x}, \mathbf{y}): i \in I\right\rangle$ be a family of atomic formulæ with parameters from $A$. Then the "projection"

$$
X=\left\{x \in A: A \models(\exists \mathbf{y}) \bigwedge_{i \in I} \varphi_{i}(x, \mathbf{y})\right\}
$$

is closed under finite meet and join.

Proof. Let $B=A \times A$; identify $A$ with the diagonal of $B$, so that is is a sublattice of $B$. For all $x=\left(x_{0}, x_{1}\right) \in B$, put $x^{+}=x_{0} \vee x_{1}$ and $x^{-}=x_{0} \wedge x_{1}$. Then it is immediate to verify that $\left(B, \wedge, \vee,{ }^{+},{ }^{-}\right)$is a projection algebra, with center $A$. Let $x_{0}$ and $x_{1}$ in $X$. Thus there are $y_{0}$ and $y_{1}$ in $A$ such that for all $i \in I, A$ satisfies $\varphi_{i}\left(x_{0}, y_{0}\right)$ and $\varphi_{i}\left(x_{1}, y_{1}\right)$, i.e. $B$ satisfies $\varphi_{i}(x, y)$ where $x=\left(x_{0}, x_{1}\right)$ and $y=\left(y_{0}, y_{1}\right)$. Therefore, by proposition 1.7, $A$ satisfies $\varphi_{i}(u, v)$ where $u=x^{+}=x_{0} \vee x_{1}$ and $v=(x \wedge y)^{+} \vee y^{-}=\left(x_{0} \wedge y_{0}\right) \vee\left(x_{1} \wedge y_{1}\right) \vee\left(y_{0} \wedge y_{1}\right)$. Therefore, $x_{0} \vee x_{1}=u \in X$, so that $X$ is closed under finite join. By lattice duality, $X$ is also closed under finite meet.

1.9. Remark. Note that in the context of corollary $1.8, A \times A$ is a reduced power of $A$. Thus, corollary 1.8 is also a consequence of Nauryzbaev's original result.

The next section will provide us with counterexamples proving among other things that there is no 3 -variable analogue of corollary 1.8 valid in every distributive lattice.

We shall now conclude this section by discussing ultracontinuous lattices. For every filter $\mathcal{F}$ on a complete lattice $A$, define $\underline{\lim }(\mathcal{F})=\underline{\lim }_{\mathcal{F}} i d_{A}$ and $\overline{\lim }(\mathcal{F})=\varlimsup_{\mathcal{l}} i d_{A}$ (see example 1.5, (3)); when $\underline{\lim }(\mathcal{F})=\varlimsup \lim (\mathcal{F})$, we denote the common value by $\lim (\mathcal{F})$. Similarly, if $f$ is a map from a set $\Omega$ to $A, \mathcal{F}$ is a filter on $\Omega$ and $\underline{\lim }_{\mathcal{F}} f=\varlimsup_{\mathcal{F}} f$, then we denote the common value by $\lim _{\mathcal{F}} f$. It is clear that $\underline{\lim }_{\mathcal{F}} f=\underline{\lim }\left(f_{*} \mathcal{F}\right)$ where $f_{*} \mathcal{F}=\left\{Y \subseteq A: f^{-1}[Y] \in \mathcal{F}\right\}:$ indeed, $\{f[X]: X \in \mathcal{F}\}$ is a filterbasis of $f_{*} \mathcal{F}$. The result is similar for $\varlimsup$ lim and lim.

Say that $A$ is ultracontinuous when for every ultrafilter $\mathcal{U}$ on $A$, we have $\underline{\lim }(\mathcal{U})=$ $\varlimsup \lim (\mathcal{U})$ (this definition is equivalent to the one in $[\mathbf{1 9}]$ by previous remark). Thus in particular, every finite lattice is ultracontinuous. The following (easy) proposition generalizes $[\mathbf{1 0}$, exercise III $3.23,(6) \Leftrightarrow(7)]$. 
1.10. Proposition. Let $A$ be a lattice. Then the following are equivalent:

(i) The interval topology of $A$ is compact Hausdorff;

(ii) $A$ is complete and ultracontinuous.

Furthermore, if (i) or (ii) is realized, then every ultrafilter $\mathcal{U}$ on $A$ has $\lim (\mathcal{U})$ as unique topological limit.

Proof. (i) $\Rightarrow$ (ii) Suppose that the interval topology on $A$ is compact. For every nonempty bounded above subset $S$ of $A$, if $T$ is the set of all upper bounds of $S$ in $A$, then the set of all closed intervals $[s, t]$ where $s \in S$ and $t \in T$ has the finite intersection property (because $A$ is a lattice), thus they have nonempty intersection; any element of this intersection is the l.u.b. of $S$ in $A$. A similar but simpler argument shows that $A$ has a least and a largest element. This proves that $A$ is complete (see also $[\mathbf{8}, \mathbf{2 5}]$ ). Let $\mathcal{U}$ be a ultrafilter on $A$; by [16, proposition VII 1.11], $\mathcal{U}$ converges to $\operatorname{both} \underline{\lim }(\mathcal{U})$ and $\overline{\lim }(\mathcal{U})$ for the interval topology; thus, if in addition the interval topology of $A$ is Hausdorff, then $\underline{\lim }(\mathcal{U})=\overline{\lim }(\mathcal{U})$, thus proving that $A$ is ultracontinuous.

(ii) $\Rightarrow$ (i) By [16, proposition VII 1.11], every ultrafilter $\mathcal{U}$ of $A$ converges to a unique element of $A$ for the interval topology, which is $\lim (\mathcal{U})$ (the last assertion of the proposition follows). By [16, proposition III 2.2], the interval topology of $A$ is compact Hausdorff.

1.11. Lemma. Let $\Omega$ be a set, let $\mathcal{F}$ be a filter on $\Omega$, let $A$ be a complete lattice and let $f$ and $g$ be maps from $\Omega$ to $A$. Equip ${ }^{\Omega} A$ with its product lattice structure. Then the following holds:

(i) If $A$ is meet-continuous, then $\underline{\lim }_{\mathcal{F}}(f \wedge g)=\underline{\lim }_{\mathcal{F}} f \wedge \underline{\lim }_{\mathcal{F}} g$;

(ii) If $A$ is join-continuous, then $\overline{\lim }_{\mathcal{F}}(f \vee g)=\overline{\lim }_{\mathcal{F}} f \vee \overline{\lim }_{\mathcal{F}} g$.

Proof. (i) A direct calculation:

$$
\begin{aligned}
\varliminf_{\mathcal{F}} f \wedge \underline{\lim }_{\mathcal{F}} g & =\bigvee_{X \in \mathcal{F}} \bigwedge f[X] \wedge \bigvee_{Y \in \mathcal{F}} \bigwedge g[Y] \\
& =\bigvee_{X, Y \in \mathcal{F}}[\bigwedge f[X] \wedge \bigwedge g[Y]] \quad \text { because } A \text { is meet-continuous } \\
& =\bigvee_{Z \in \mathcal{F}}[\bigwedge f[Z] \wedge \bigwedge g[Z]] \quad \text { because } \mathcal{F} \text { is closed under finite intersection } \\
& =\bigvee_{Z \in \mathcal{F}} \bigwedge(f \wedge g)[Z] \\
& =\underline{\lim }_{\mathcal{F}}(f \wedge g) .
\end{aligned}
$$

The proof of (ii) is just the dual of the proof of (i).

1.12. Lemma. Let $A$ be a complete, meet-continuous, join-continuous ultracontinuous lattice. Then the meet and join operations from $A \times A$ (endowed with the square of the interval topology of $A$ ) to $A$ (endowed with the interval topology) are continuous. 
Proof. First, note that since $A$ has a least and a largest element, the interval topology of $A \times A$ is identical to the square of the interval topology of $A$. Furthermore, it is trivial that $A \times A$ is itself a complete, meet-continuous, join-continuous and ultracontinuous lattice; thus, endowed with its interval topology, it is compact Hausdorff (we use proposition 1.10). Therefore, by [16, lemma III 2.3], it suffices to prove that the meet and the join operations (denote them respectively by $\delta$ and $\sigma$ ) preserve limits of ultrafilters. Let $p: A \times A \rightarrow$ $A,(x, y) \mapsto x$ and $q: A \times A \rightarrow A,(x, y) \mapsto y$. A direct computation shows that for every filter $\mathcal{F}$ on $A \times A$, we have $\underline{\lim }_{\mathcal{F}} p=p(\underline{\lim }(\mathcal{F}))$ and $\underline{\lim }_{\mathcal{F}} q=q(\underline{\lim }(\mathcal{F}))$, and similarly for $\varlimsup$ lim and thus also for lim. Using lemma 1.11, we obtain that for every ultrafilter $\mathcal{U}$ on $A \times A$, we have

$$
\begin{aligned}
\lim _{\mathcal{U}} \delta=\lim _{\mathcal{U}}(p \wedge q) & =\underline{\lim }_{\mathcal{U}}(p \wedge q) \\
& =\underline{\lim }_{\mathcal{U}} p \wedge \underline{\lim }_{\mathcal{U}} q \\
& =\lim _{\mathcal{U}} p \wedge \lim _{\mathcal{U}} q \\
& =p(\lim (\mathcal{U})) \wedge q(\lim (\mathcal{U})) \\
& =\delta(\lim (\mathcal{U})),
\end{aligned}
$$

and similarly, $\lim _{\mathcal{U}} \sigma=\sigma(\lim (\mathcal{U}))$. This concludes the proof.

Now we can state the

1.13. Proposition. Let $A$ be a lattice. Then the following are equivalent:

(i) The interval topology of $A$ makes $A$ into a compact Hausdorff topological lattice;

(ii) $A$ is complete, meet-continuous, join-continuous and ultracontinuous.

Proof. (i) $\Rightarrow$ (ii) If (i) holds, then $A$ is equationally compact, thus [12] complete, meetcontinuous and join-continuous; furthermore, by proposition 1.10, $A$ is ultracontinuous.

(ii) $\Rightarrow$ (i) results froms proposition 1.10 and lemma 1.12 .

1.14. Example. This example will illustrate the fact that the conditions of proposition 1.13 (ii) are not redundant, even for distributive lattices. Endow $A=(\omega \times \omega) \cup\{\infty\}$ with the partial ordering $\leq$ extending the natural ordering of $\omega \times \omega$ with $\infty$ as largest element. It is easy to verify that $A$ is a complete ultracontinuous distributive lattice. It is well-founded for $\leq$ thus join-continuous. It is not meet-continuous because if we put $a=(1,0)$ and $b=(0,1)$, then $a \wedge \bigvee_{n \in \mathbb{N}} n b=a>0$ while $\bigvee_{n \in \mathbb{N}}(a \wedge n b)=0$

Using [16, theorem VII 1.14], one can immediately deduce from proposition 1.13 the following

1.15. Corollary. Let $A$ be a distributive lattice. Then the following are equivalent:

(i) The interval topology makes $A$ into a compact Hausdorff topological lattice;

(ii) $A$ is an ultracontinuous bi-frame;

(iii) $A$ is completely distributive. 
Proposition 1.13 is a strengthening of [19, theorem 1] since it shows that complete, meet-continuous, join-continuous and ultracontinuous lattices are not only equationally compact, but even topologically compact. Corollary 1.15 unifies $[\mathbf{1 7}$, theorem 1] and [19, theorem 2(b)] (where it is proved that every bi-frame without infinite antichains is ultracontinuous, thus equationally compact) and [16, corollary VII 1.11] (where it is proved that every completely distributive lattice is ultracontinuous). In particular, it implies, using $[\mathbf{1 9}$, theorem 2], that every bi-frame that is either weakly atomic or without infinite antichains is completely distributive.

\section{§2. THE FUNDAMENTAL FINITE EXAMPLES.}

We start with the following example.

2.1. Example. Let $P_{0}$ be the 8-element set $\left\{a, b, c, d, a^{\prime}, b^{\prime}, c^{\prime}, d^{\prime}\right\}$, endowed with the partial ordering represented below:

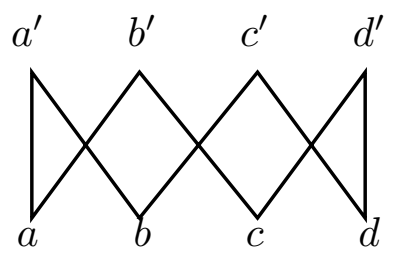

This means that $a<a^{\prime}, b^{\prime}$ and $b<a^{\prime}, c^{\prime}$ and $c<b^{\prime}, d^{\prime}$ and $d<c^{\prime}, d^{\prime}$ and that $<$ does not contain any other pair. Let $\mathcal{E}_{0}$ be the (finite) distributive lattice of all lower subsets of $P_{0}$. Define elements of $\mathcal{E}_{0}$ the following way:

$$
\begin{aligned}
& U=\downarrow\left\{a^{\prime}\right\}, \quad U^{\prime}=\downarrow\left\{d^{\prime}\right\}, \quad V=\downarrow\left\{b^{\prime}\right\}, \quad V^{\prime}=\downarrow\left\{c^{\prime}\right\} ; \\
& A=U \cup U^{\prime}, \quad B=V \cup V^{\prime}, \quad C=U \cup V, \quad D=U^{\prime} \cup V^{\prime}
\end{aligned}
$$

Let $\varphi(\mathbf{x}, \mathbf{y}, \mathbf{z})$ be the following formula of the language $(\wedge, \vee)$ (by writing as usual $\mathbf{x} \leq \mathbf{y}$ instead of $\mathbf{x} \wedge \mathbf{y}=\mathbf{x}$ ), with parameters in $\mathcal{E}_{0}$ :

$$
\mathbf{z}=\mathbf{x} \vee \mathbf{y} \text { and } \mathbf{x} \wedge \mathbf{y}=\emptyset \text { and } \mathbf{x} \leq C \text { and } \mathbf{y} \leq D
$$

It is clear that $\mathcal{E}_{0}$ satisfies $\varphi\left(U, U^{\prime}, A\right)$ and $\varphi\left(V, V^{\prime}, B\right)$. Now, suppose that $\mathcal{E}_{0}$ satisfies $\varphi(X, Y, A \cup B)$ for some $X, Y$ in $\mathcal{E}_{0}$. Since $A \cup B=P_{0}$, this means that $X, Y$ satisfy the following system:

$$
\left\{\begin{array}{l}
X \cup Y=P_{0} \\
X \cap Y=\emptyset \\
X \subseteq C \\
Y \subseteq D
\end{array}\right.
$$

Thus $Y=\complement\left[X\right.$, whence $Y$ is both a lower subset and an upper subset of $P_{0}$. But it is clear that $P_{0}$ is connected, thus $Y=\emptyset$ or $Y=P_{0}$, thus $X=P_{0}$ or $Y=P_{0}$, which contradicts (2.1). Therefore, we have proved the following fact: 
$\varphi$ is a conjunction of atomic formulæ with parameters from $\mathcal{E}_{0}, \mathcal{X}=\left\{Z \in \mathcal{E}_{0}: \mathcal{E}_{0} \models\right.$ $(\exists \mathbf{x}, \mathbf{y}) \varphi(\mathbf{x}, \mathbf{y}, Z)\}$ has $A$ and $B$ as elements, but not $A \cup B$.

Therefore, as promised, corollary 1.8 does not generalize fully to the 3 -variable case.

Note that $A \cap B$ belongs to $\mathrm{X}$, and in fact, this example does not destroy completely any hope to generalize Nauryzbaev's formula to the 3-variable case. Say that a proper term is a lattice combination of terms of the form $\left(\bigwedge_{i \in I} \mathbf{x}_{i}\right)^{+}$or $\left(\bigvee_{i \in I} \mathbf{x}_{i}\right)^{-}$. Example 2.1 shows certainly that there are no proper terms $t_{0}, t_{1}, t_{2}$ such that $t_{0}\left(\mathbf{x}_{0}, \mathbf{x}_{1}, \mathbf{x}_{2}\right)=\mathbf{x}_{0}^{+}$and for every projection algebra $A$ and every formula $\varphi\left(\mathbf{x}_{0}, \mathbf{x}_{1}, \mathbf{x}_{2}\right)$ with parameters from $Z(A), A$ satisfies

$$
\left(\forall \mathbf{x}_{0}, \mathbf{x}_{1}, \mathbf{x}_{2}\right)\left[\varphi\left(\mathbf{x}_{0}, \mathbf{x}_{1}, \mathbf{x}_{2}\right) \Rightarrow \varphi\left(t_{0}\left(\mathbf{x}_{0}, \mathbf{x}_{1}, \mathbf{x}_{2}\right), t_{1}\left(\mathbf{x}_{0}, \mathbf{x}_{1}, \mathbf{x}_{2}\right), t_{2}\left(\mathbf{x}_{0}, \mathbf{x}_{1}, \mathbf{x}_{2}\right)\right)\right]
$$

In the language of monadic algebras, this implies that countable projection algebras are not rich (see [15, page 77 , theorem 2]). Still, hope remains that one could satisfy (2.2) if one drops the assumption $t_{0}\left(\mathbf{x}_{0}, \mathbf{x}_{1}, \mathbf{x}_{2}\right)=\mathbf{x}_{0}^{+}$. Can this be done? A more involved algebraic study of projection algebras (which we shall not need in this paper) shows that again, the answer to this new question is no. A decisive byproduct of the proof is the finite partially ordered set that we are going to describe now.

2.2. Definition. $P$ is the 6 -element set $\left\{p_{0}, p_{1}, p_{2}, q_{0}, q_{1}, q_{2}\right\}$ endowed with the partial ordering whose graph is the following:

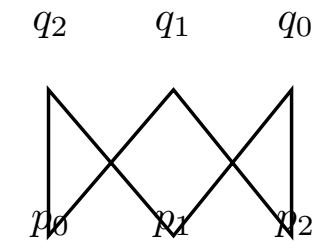

Hence, for all $p, q$ in $P, p<q$ if and only if there exist $i, j<3$ such that $i \neq j$ and $p=p_{i}$ and $q=q_{j}$. We shall fix $P$ throughout sections 2 to 4 .

2.3. Lemma. Let $f: P \rightarrow P$ be an increasing map such that $(\forall i<3)\left(f\left(q_{i}\right) \leq q_{i}\right)$. Then $f$ is the identity map.

Proof. Let $i<3$, let $j, k$ such that $3=\{i, j, k\}$. Then $f\left(p_{i}\right) \leq f\left(q_{j}\right), f\left(q_{k}\right)$, thus, by assumption, $f\left(p_{i}\right) \leq q_{j}, q_{k}$, so that $f\left(p_{i}\right)=p_{i}$. Similarly, $f\left(p_{j}\right), f\left(p_{k}\right) \leq f\left(q_{i}\right)$ thus $p_{j}, p_{k} \leq f\left(q_{i}\right)$, whence $f\left(q_{i}\right)=q_{i}$.

For every set $D$ and every $d \in D$, denote by $\pi_{d}^{D}$ the $d$ th projection from ${ }^{D} P$ to $P$, i.e. the map ${ }^{D} P \rightarrow P,\left\langle x_{i}: i \in D\right\rangle \mapsto x_{d}$.

The most important lemma of this section (and perhaps of the whole paper) is the following 
2.4. Lemma. Let $f: P \times P \rightarrow P$ be an increasing map satisfying $(\forall i<3)\left(f\left(q_{i}, q_{i}\right) \leq q_{i}\right)$. Then $f$ is a projection, i.e. $f=\pi_{0}^{2}$ or $f=\pi_{1}^{2}$.

Proof. We prove the lemma in a series of claims.

Claim 1. $(\forall x \in P)(f(x, x)=x)$.

Proof of claim. It suffices to apply lemma 2.3 to the map $x \mapsto f(x, x)$ a Claim 1 .

Claim 2. For all $i, j<3$ such that $i \neq j$, both $f\left(p_{i}, q_{j}\right)$ and $f\left(q_{j}, p_{i}\right)$ belong to $\left\{p_{i}, q_{j}\right\}$.

Proof of claim. We have $p_{i}=f\left(p_{i}, p_{i}\right) \leq f\left(p_{i}, q_{j}\right) \leq f\left(q_{j}, q_{j}\right)=q_{j}$, and similarly for $f\left(q_{j}, p_{i}\right)$. The conclusion follows.

- Claim 2.

Claim 3. For all $i, j, k$ such that $3=\{i, j, k\}$, we have $f\left(q_{i}, q_{j}\right) \in\left\{p_{k}, q_{i}, q_{j}\right\}$.

Proof of claim. We have $p_{k}=f\left(p_{k}, p_{k}\right) \leq f\left(q_{i}, q_{j}\right)$, whence $f\left(q_{i}, q_{j}\right) \in\left\{p_{k}, q_{i}, q_{j}\right\}$.

- Claim 3.

Claim 4. For all $i, j<3$, we have $f\left(p_{i}, p_{j}\right) \in\left\{p_{i}, p_{j}\right\}$.

Proof of claim. For $i=j$ it results from claim 1 . Suppose that $i \neq j$, and let $\{k\}=$ $3 \backslash\{i, j\}$. Then we have $f\left(p_{i}, p_{j}\right) \leq f\left(q_{k}, q_{k}\right)=q_{k}$ and, using claim $3, f\left(p_{i}, p_{j}\right) \leq f\left(q_{j}, q_{i}\right) \in$ $\left\{p_{k}, q_{i}, q_{j}\right\}$. It follows that $f\left(p_{i}, p_{j}\right) \in\left\{p_{i}, p_{j}, q_{k}\right\} \cap\left\{p_{k}, p_{i}, p_{j}, q_{i}, q_{j}\right\}=\left\{p_{i}, p_{j}\right\}$. Claim 4 .

Claim 5. For all $i, j<3$, we have $f\left(q_{i}, q_{j}\right) \in\left\{q_{i}, q_{j}\right\}$.

Proof of claim. For $i=j$ it results from claim 1 . So suppose that $i \neq j$, and let $\{k\}=3 \backslash\{i, j\}$. Then we have $f\left(q_{i}, q_{j}\right) \in\left\{p_{k}, q_{i}, q_{j}\right\}$ by claim 3, and, using claim 4 , $f\left(q_{i}, q_{j}\right) \geq f\left(p_{j}, p_{i}\right) \in\left\{p_{i}, p_{j}\right\}$. The result follows.

- Claim 5.

Claim 6. $(\forall x, y \in P)(f(x, y) \in\{x, y\})$.

Proof of claim. According to claims 1-5, it suffices to prove the claim for all ordered pairs $\left(p_{i}, q_{i}\right)$ or $\left(q_{i}, p_{i}\right)$ for $i<3$. Let $j, k$ such that $3=\{i, j, k\}$. Suppose that $f\left(p_{i}, q_{i}\right) \notin$ $\left\{p_{i}, q_{i}\right\}$. We have $f\left(p_{i}, q_{i}\right) \leq f\left(q_{j}, q_{i}\right) \in\left\{q_{j}, q_{i}\right\}$ and $f\left(p_{i}, q_{i}\right) \leq f\left(q_{k}, q_{i}\right) \in\left\{q_{k}, q_{i}\right\}$ (we use claim 5). Thus $f\left(p_{i}, q_{i}\right) \in\left\{p_{0}, p_{1}, p_{2}, q_{i}\right\}$, thus, by assumption, $f\left(p_{i}, q_{i}\right) \in\left\{p_{j}, p_{k}\right\}$. But $f\left(p_{i}, q_{i}\right) \geq f\left(p_{i}, p_{j}\right) \in\left\{p_{i}, p_{j}\right\}$ and $f\left(p_{i}, q_{i}\right) \geq f\left(p_{i}, p_{k}\right) \in\left\{p_{i}, p_{k}\right\}$ (use claim 4); the first possibility contradicts $f\left(p_{i}, q_{i}\right)=p_{k}$ and the second possibility contradicts $f\left(p_{i}, q_{i}\right)=$ $p_{j}$. So we obtain a contradiction, whence $f\left(p_{i}, q_{i}\right) \in\left\{p_{i}, q_{i}\right\}$. The proof for $f\left(q_{i}, p_{i}\right)$ is similar.

- Claim 6.

In particular, it results from claim 5 that $f\left(q_{0}, q_{1}\right) \in\left\{q_{0}, q_{1}\right\}$. Suppose from now on that $f\left(q_{0}, q_{1}\right)=q_{0}$.

Put $X=\{(x, y) \in P \times P: f(x, y)=x\}$. We shall prove that $X=P \times P$.

Note that the diagonal $\Delta=\{(x, x): x \in P\}$ is contained in $X$, and that $\left(q_{0}, q_{1}\right) \in X$ by assumption. Define binary relations $R$ and $S$ on $P \times P$ by

$$
\begin{aligned}
& (x, y) R\left(x^{\prime}, y^{\prime}\right) \Leftrightarrow\left(x \leq x^{\prime} \text { and } y \leq y^{\prime} \text { and } x \not \leq y^{\prime}\right), \\
& (x, y) S\left(x^{\prime}, y^{\prime}\right) \Leftrightarrow\left(x \leq x^{\prime} \text { and } y \leq y^{\prime} \text { and } y \not \leq x^{\prime}\right) .
\end{aligned}
$$


Claim 7. $X$ is both a $R$-upper set and a $S$-lower set.

Proof of claim. Let $(x, y)$ and $\left(x^{\prime}, y^{\prime}\right)$ in $P \times P$. Suppose that $(x, y) R\left(x^{\prime}, y^{\prime}\right)$ and $(x, y) \in$ $X$. Then we have $x=f(x, y) \leq f\left(x^{\prime}, y^{\prime}\right) \in\left\{x^{\prime}, y^{\prime}\right\}$; but since $x \not \leq y^{\prime}$, the only possibility which remains is $f\left(x^{\prime}, y^{\prime}\right)=x^{\prime}$, i.e. $\left(x^{\prime}, y^{\prime}\right) \in X$. This proves that $X$ is a $R$-upper set.

Similarly, let $(x, y)$ and $\left(x^{\prime}, y^{\prime}\right)$ in $P \times P$. Suppose that $(x, y) S\left(x^{\prime}, y^{\prime}\right)$ and $\left(x^{\prime}, y^{\prime}\right) \in X$. Then we have $x^{\prime}=f\left(x^{\prime}, y^{\prime}\right) \geq f(x, y) \in\{x, y\}$; but since $x^{\prime} \geq y$, the only possibility which remains is $f(x, y)=x$, i.e. $(x, y) \in X$. This proves that $X$ is a $S$-lower set.

- Claim 7.

Now we prove that $X=P \times P$, by proving that $P \times P \backslash\left(\Delta \cup\left\{\left(q_{0}, q_{1}\right)\right\}\right) \subseteq X$. The reader may find it helpful to plot all the elements of $P \times P \backslash \Delta$ on an array and check them one after the other once they are found to lie in $X$. Thus the picture at the beginning looks like this:

\begin{tabular}{|l|l|l|l|l|l|l|l|l|l|l|l|}
\hline$\left(p_{0}, p_{1}\right)$ & & $\left(p_{1}, p_{0}\right)$ & & $\left(p_{2}, p_{0}\right)$ & & $\left(q_{0}, p_{0}\right)$ & & $\left(q_{1}, p_{0}\right)$ & & $\left(q_{2}, p_{0}\right)$ & \\
\hline$\left(p_{0}, p_{2}\right)$ & & $\left(p_{1}, p_{2}\right)$ & & $\left(p_{2}, p_{1}\right)$ & & $\left(q_{0}, p_{1}\right)$ & & $\left(q_{1}, p_{1}\right)$ & & $\left(q_{2}, p_{1}\right)$ & \\
\hline$\left(p_{0}, q_{0}\right)$ & & $\left(p_{1}, q_{0}\right)$ & & $\left(p_{2}, q_{0}\right)$ & & $\left(q_{0}, p_{2}\right)$ & & $\left(q_{1}, p_{2}\right)$ & & $\left(q_{2}, p_{2}\right)$ & \\
\hline$\left(p_{0}, q_{1}\right)$ & & $\left(p_{1}, q_{1}\right)$ & & $\left(p_{2}, q_{1}\right)$ & & $\left(q_{0}, q_{1}\right)$ & $\checkmark$ & $\left(q_{1}, q_{0}\right)$ & & $\left(q_{2}, q_{0}\right)$ & \\
\hline$\left(p_{0}, q_{2}\right)$ & & $\left(p_{1}, q_{2}\right)$ & & $\left(p_{2}, q_{2}\right)$ & & $\left(q_{0}, q_{2}\right)$ & & $\left(q_{1}, q_{2}\right)$ & & $\left(q_{2}, q_{1}\right)$ & \\
\hline
\end{tabular}

Now we proceed:

- If $x \in\left\{q_{0}, p_{1}, p_{2}\right\}$ and $y \in\left\{q_{1}, p_{0}\right\}$, then $(x, y) S\left(q_{0}, q_{1}\right) \in X$, thus $(x, y) \in X$ (5 new elements).

- If $y \in\left\{p_{0}, q_{1}\right\}$, then $X \ni\left(p_{1}, p_{0}\right) R\left(q_{2}, y\right)$, thus $\left(q_{2}, y\right) \in X$ (2 new elements).

- $\left(p_{1}, p_{2}\right) S\left(p_{1}, q_{1}\right) \in X$, thus $\left(p_{1}, p_{2}\right) \in X$ (1 new element).

- If $x \in\left\{p_{2}, q_{0}, q_{1}\right\}$ and $y \in\left\{p_{0}, q_{2}\right\}$, then $X \ni\left(p_{2}, p_{0}\right) R(x, y)$, thus $(x, y) \in X$ (4 new elements).

$-\left(p_{2}, p_{1}\right) S\left(p_{2}, q_{2}\right) \in X$, thus $\left(p_{2}, p_{1}\right) \in X$ (1 new element $)$.

- $\left(p_{1}, q_{2}\right) S\left(q_{0}, q_{2}\right) \in X$, thus $\left(p_{1}, q_{2}\right) \in X$ (1 new element $)$.

- If $x \in\left\{q_{1}, p_{0}\right\}$ and $y \in\left\{q_{2}, p_{1}\right\}$, then $(x, y) S\left(q_{1}, q_{2}\right) \in X$, thus $(x, y) \in X$ (3 new elements).

- If $x \in\left\{q_{2}, p_{0}\right\}$ and $y \in\left\{q_{1}, p_{2}\right\}$, then $(x, y) S\left(q_{2}, q_{1}\right) \in X$, thus $(x, y) \in X$ (3 new elements).

- If $x \in\left\{p_{0}, q_{1}, q_{2}\right\}$ and $y \in\left\{p_{1}, q_{0}\right\}$, then $X \ni\left(p_{0}, p_{1}\right) R(x, y)$, thus $(x, y) \in X$ (4 new elements).

- $X \ni\left(p_{0}, p_{2}\right) R\left(q_{1}, p_{2}\right)$, thus $\left(q_{1}, p_{2}\right) \in X$ (1 new element $)$.

$-\left(p_{1}, q_{0}\right) S\left(q_{2}, q_{0}\right) \in X$, thus $\left(p_{1}, q_{0}\right) \in X$ (1 new element $)$.

$-\left(p_{2}, q_{0}\right) S\left(q_{1}, q_{0}\right) \in X$, thus $\left(p_{2}, q_{0}\right) \in X$ (1 new element $)$. 
- $X \ni\left(p_{2}, p_{1}\right) R\left(q_{0}, p_{1}\right)$, thus $\left(q_{0}, p_{1}\right) \in X$ (1 new element $)$.

- $X \ni\left(p_{1}, p_{2}\right) R\left(q_{0}, p_{2}\right)$, thus $\left(q_{0}, p_{2}\right) \in X$ (1 new element $)$.

Hence, $X=P \times P$, so that $f=\pi_{0}^{2}$.

Suppose now that $f\left(q_{0}, q_{1}\right)=q_{1}$. Let $\tau$ be the involutive automorphism of $P$ exchanging $q_{0}$ and $q_{1}$ : that is, $\tau\left(q_{0}\right)=q_{1}, \tau\left(q_{1}\right)=q_{0}, \tau\left(q_{2}\right)=q_{2}, \tau\left(p_{0}\right)=p_{1}, \tau\left(p_{1}\right)=p_{0}$, $\tau\left(p_{2}\right)=p_{2}$. Let $g: P \times P \rightarrow P$ defined by $g(x, y)=\tau f(\tau y, \tau x)$. Then $g$ is increasing, $g\left(q_{i}, q_{i}\right)=q_{i}$ for all $i<3$ and $g\left(q_{0}, q_{1}\right)=q_{0}$. By the previous result, $g=\pi_{0}^{2}$. It follows that $f=\pi_{1}^{2}$.

Actually, this is a particular case of a more general (and non-trivial) result, kindly pointed to us by Maurice Pouzet, due to E. Corominas [7]; but our proof presented here is self-contained.

Now we can construct a further example.

2.5. Example. For all $n$ in $\omega \backslash\{0\}$, let $\mathbb{E}_{n}$ be the (finite) distributive lattice of all lower subsets of ${ }^{n} P$. For all $i<3$, put $A_{i}=\downarrow\left\{q_{i}\right\} \in \mathbb{E}_{1}$. Let $\varphi\left(\mathbf{x}_{0}, \mathbf{x}_{1}, \mathbf{x}_{2}\right)$ be the following conjunction of atomic formulæ of the language $(\wedge, \vee)$, with parameters from $\mathbb{E}_{2}$ :

$\mathbf{x}_{0} \wedge \mathbf{x}_{1} \wedge \mathbf{x}_{2}=\emptyset$ and $\mathbf{x}_{0} \vee \mathbf{x}_{1} \vee \mathbf{x}_{2}=P \times P$ and $A_{0} \times A_{0} \leq \mathbf{x}_{0}$ and $A_{1} \times A_{1} \leq \mathbf{x}_{1}$ and $A_{2} \times A_{2} \leq \mathbf{x}_{2}$.

Let $X_{0}, X_{1}, X_{2}$ in $\mathbb{E}_{2}$ such that $\mathbb{E}_{2}$ satisfies $\varphi\left(X_{0}, X_{1}, X_{2}\right)$. Since $X_{0} \cap X_{1} \cap X_{2}=\emptyset$ and $X_{0} \cup X_{1} \cup X_{2}=P \times P$, it is easy to verify that there exists a unique map $f: P \times P \rightarrow P$ such that for all $(x, y) \in P \times P$ and all $i<3$, we have $f(x, y) \leq q_{i} \Leftrightarrow(x, y) \in X_{i}$. Since $A_{i} \times A_{i} \subseteq X_{i}$ for all $i<3$, we have $f\left(q_{i}, q_{i}\right) \leq q_{i}$. Since the $X_{i}$ 's are lower subsets of $P \times P, f$ is increasing. By lemma 2.4, $f$ is a projection. If for example $f=\pi_{0}^{2}$, then for all $i<3$ and all $(x, y) \in P \times P$, we have

$$
\begin{aligned}
(x, y) \in X_{i} & \Leftrightarrow f(x, y) \leq q_{i} \\
& \Leftrightarrow x \leq q_{i} \\
& \Leftrightarrow x \in A_{i},
\end{aligned}
$$

so that $X_{i}=A_{i} \times P$ for all $i<3$. Similarly, if $f=\pi_{1}^{2}$, then for all $i<3, X_{i}=P \times A_{i}$. So we have proved the following

Fact. The only triples $\left(X_{0}, X_{1}, X_{2}\right)$ of elements of $\mathbb{E}_{2}$ such that $\mathbb{E}_{2}$ satisfies $\varphi\left(X_{0}, X_{1}, X_{2}\right)$ are $\left(A_{0} \times P, A_{1} \times P, A_{2} \times P\right)$ and $\left(P \times A_{0}, P \times A_{1}, P \times A_{2}\right)$.

In particular, the only elements $Z$ of $\mathbb{E}_{2}$ such that $\mathbb{E}_{2}$ satisfies $(\exists \mathbf{x}, \mathbf{y}) \varphi(\mathbf{x}, \mathbf{y}, Z)$ are $W=A_{2} \times P$ and $W^{\prime}=P \times A_{2}$. But if $\sigma$ is the involutive automorphism of $\mathbb{E}_{2}$ corresponding to the map $(x, y) \mapsto(y, x)$, then $\sigma$ exchanges $W$ and $W^{\prime}$ and all parameters of $\varphi$ are invariant under $\sigma$. One can easily see that this implies that there cannot be any generalization of Nauryzbaev's formula to the case of 3 variables.

2.6. Remark. In [15, page 77 , theorem 2], it is proved that every countable monadic algebra is "rich", so that in particular, its center is a retract of the whole algebra. By 
contrast, example 2.5 provides us with a finite projection algebra $A$ such that $Z(A)$ is not a retract of $A$. Indeed, let $G=\{1, \sigma\}$ act on $\mathbb{E}_{2}$; associate a projection algebra to this action as in example $1.5(2)$, and let $\mathbb{E}^{*}$ be the center of this projection algebra, i.e. the sublattice of all fixed points of $\sigma$ in $\mathbb{E}_{2}$. Then $\mathbb{E}_{2}$ satisfies the statement $\left(\exists \mathbf{x}_{0}, \mathbf{x}_{1}, \mathbf{x}_{2}\right) \varphi\left(\mathbf{x}_{0}, \mathbf{x}_{1}, \mathbf{x}_{2}\right)$ (all parameters of $\varphi$ lie in $\mathbb{E}^{*}$ and $\varphi$ is a conjunction of atomic formulæ), while $\mathbb{E}^{*}$ does not satisfy this statement.

\section{$\S$ 3. CASE OF $\sigma$-BI-FRAMES.}

Since the proof of failure of 3 -variable countable equational compactness for $\sigma$-biframes is easier than the proof for bi-frames (and also prepares for it), we will concentrate on it in this section. We first need a slight improvement of lemma 2.4.

3.1. Lemma. Let $f:{ }^{3} P \rightarrow P$ be an increasing map such that for all $i<3, f\left(q_{i}, q_{i}, q_{i}\right) \leq$ $q_{i}$. Then $f$ is a projection, i.e. $f=\pi_{k}^{3}$ for some $k<3$.

Proof. We argue by cases.

Case 1. $f(x, x, y)=x$ and $f(x, y, y)=y$ identically.

Let $z$ in $P$, and let $f^{z}: P \times P \rightarrow P,(x, y) \mapsto f(x, y, z)$. Then $f^{z}$ is increasing and for all $x$ in $P, f^{z}(x, x)=x$, thus, by lemma 2.4, $f^{z}$ is a projection. Suppose that $f^{z}=\pi_{0}^{2}$. Then for all $x$ in $P$, we have $f(x, z, z)=f^{z}(x, z)=x$, contradicts for $x \neq z$ the hypothesis that $f(x, z, z)=z$. Thus $f^{z}=\pi_{1}^{2}$. This holds for all $z$ in $P$; therefore, $f(x, y, z)=y$ identically. This concludes case 1 .

Case 2. $f(x, x, y)=x$ and $f(x, y, y)=x$ identically.

Let $z$ in $P$, and let $f^{z}: P \times P \rightarrow P,(x, y) \mapsto f(x, y, z)$. Then $f^{z}$ is increasing and for all $x$ in $P, f^{z}(x, x)=x$, thus, by lemma $2.4, f^{z}$ is a projection. Suppose that $f^{z}=\pi_{1}^{2}$. Then for all $x$ in $P$, we have $f(x, z, z)=f^{z}(x, z)=z$, contradicts for $x \neq z$ the hypothesis that $f(x, z, z)=x$. Thus $f^{z}=\pi_{0}^{2}$. This holds for all $z$ in $P$; therefore, $f(x, y, z)=x$ identically. This concludes case 2 .

But by lemma $2.4, f(x, y, y)$ is either $x$ identically or $y$ identically. Thus cases 1 and 2 cover all cases where $f(x, x, y)=x$ identically. But up to permutation of the variables, this case is equivalent to each of both cases where $f(x, y, x)=x$ identically, and $f(y, x, x)=x$ identically, which therefore are also solved.

Thus, by lemma 2.4, it remains to solve the following case:

Case 3. $f(x, x, y)=f(x, y, x)=f(y, x, x)=y$ identically.

Then for all $x, y$ in $P, f(x, x, y)=y$ and $f(y, x, y)=x$. Take $x$ and $y$ such that $x<y$. Since $(x, x, y) \leq(y, x, y)$ and $f$ is increasing, we obtain $y \leq x$, a contradiction.

For every element $x$ of $P$ and every set $T$, denote by $x \cdot T$ the constant function defined on $T$ with value $x$.

Now we are ready to state the following

3.2. Theorem. Let $D$ be an arbitrary nonempty set, let $f:{ }^{D} P \rightarrow P$ be an increasing map such that for all $i<3, f\left(q_{i} \cdot D\right) \leq q_{i}$. Let $\mathcal{U}^{f}=\{X \subseteq D:(\forall x, y \in P)(f(x \cdot X \cup y \cdot \complement X)=x)\}$. 
Then $\mathcal{U}^{f}$ is a ultrafilter on $D$, and for all $s \in{ }^{D} P$, we have $f(s)=\lim _{\mathcal{U}^{f}} s$. In particular, if $D$ is finite, then $f$ is a projection.

Proof. For every subset $X$ of $D$, let $f_{X}: P \times P \rightarrow P,(x, y) \mapsto f(x \cdot X \cup y \cdot \complement X)$. By lemma 2.4, either $f_{X}(x, y)=x$ identically (and thus $X \in \mathcal{U}^{f}$ by definition of $\mathcal{U}^{f}$ ), or $f_{X}(x, y)=y$ identically (and then $\complement X \in \mathcal{U}^{f}$ by definition of $\mathcal{U}^{f}$ ). Thus for every $X \subseteq D, X \notin \mathcal{U}^{f}$ if and only if $\complement X \in \mathcal{U}^{f}$. Using lemma 2.3 , we see that $f(x \cdot D)=x$ identically, thus $D \in \mathcal{U}^{f}$. To prove that $\mathcal{U}^{f}$ is a ultrafilter, it remains to prove that $\mathcal{U}^{f}$ is closed under finite intersection.

So let $X, Y$ be elements of $\mathcal{U}^{f}$. Suppose that $X \cap Y \notin \mathcal{U}^{f}$. Define maps $g$ and $h$ from ${ }^{3} P$ to $P$ by

$$
(\forall x, y, z \in P)\left\{\begin{array}{l}
g(x, y, z)=f(x \cdot(X \cap Y) \cup y \cdot(X \backslash Y) \cup z \cdot \complement X) \\
h(x, y, z)=f(x \cdot(X \cap Y) \cup y \cdot(Y \backslash X) \cup z \cdot \complement Y)
\end{array}\right.
$$

By lemma 3.1, $g$ and $h$ are projections. If $g(x, y, z)=x$ identically, then, taking $y=z$, one obtains that $X \cap Y \in \mathcal{U}^{f}$, a contradiction. Furthermore, for all $x, z$ in $P$, $g(x, x, z)=f(x \cdot X \cup z \cdot \complement X)=x$ thus $g$ cannot be the projection on the last coordinate either. It follows that $g(x, y, z)=y$ identically. Similarly, $h(x, y, z)=y$ identically.

Now, fix $x$ in $P$. Define $g_{x}$ and $h_{x}$ by

$$
\begin{aligned}
& g_{x}:{ }^{3} P \rightarrow P,(y, u, v) \mapsto f(x \cdot(X \cap Y) \cup y \cdot(X \backslash Y) \cup u \cdot(Y \backslash X) \cup v \cdot(\complement X \cap \complement Y)), \\
& h_{x}:{ }^{3} P \rightarrow P,(y, u, v) \mapsto f(x \cdot(X \cap Y) \cup y \cdot(Y \backslash X) \cup u \cdot(X \backslash Y) \cup v \cdot(\complement X \cap \complement Y)) .
\end{aligned}
$$

Thus $g_{x}$ and $h_{x}$ are increasing, and since $g=h=\pi_{1}^{2}, g_{x}(y, y, y)=h_{x}(y, y, y)=y$ identically. By lemma 3.1, $g_{x}$ and $h_{x}$ are projections. Again since $g=h=\pi_{1}^{2}$, the only possibility is that $g_{x}(y, u, v)=y$ and $h_{x}(y, u, v)=y$ identically (otherwise, taking $u=v$ would contradict the fact that $g=h=\pi_{1}^{2}$ ). But this holds for all $x$. Therefore, for all $x$, $y, u, v$ in $P$, we have

$$
\begin{aligned}
& f(x \cdot(X \cap Y) \cup y \cdot(X \backslash Y) \cup u \cdot(Y \backslash X) \cup v \cdot(\complement X \cap \complement Y))=y, \\
& f(x \cdot(X \cap Y) \cup y \cdot(Y \backslash X) \cup u \cdot(X \backslash Y) \cup v \cdot(\complement X \cap \complement Y))=y .
\end{aligned}
$$

But exchanging $y$ and $u$ in the second identity contradicts the first identity.

It follows that $X \cap Y \in \mathcal{U}^{f}$, which completes the proof that $\mathcal{U}^{f}$ is a ultrafilter on $D$.

Now, to conclude, we distinguish two cases.

Case 1. $D$ is finite.

Then $\mathcal{U}^{f}$ is principal, i.e. $\mathcal{U}^{f}=(a)=\{X \subseteq D: a \in X\}$ for some $a \in D$. We prove by induction on $|D|$ that for all $s$ in ${ }^{D} P$, we have $f(s)=s(a)$. It is true for $|D|=1$ or $|D|=2$ by lemmas 2.3 and 2.4. So suppose that the result has been proved at every stage $<|D|$ with $|D| \geq 3$. Let $a \in D$ such that $\mathcal{U}^{f}=(a)$, and let $b$ and $c$ be two distinct elements of $D \backslash\{a\}$. 
Claim 1. For all $d \in D \backslash\{a\}$, let $f_{d}$ be the map defined by

$$
f_{d}:{ }^{D \backslash\{a\}} P \rightarrow P, s \mapsto f(s \cup s(d) \cdot\{a\}) .
$$

Then for all $s \in{ }^{D \backslash\{a\}} P$, we have $f_{d}(s)=s(d)$.

Proof of claim. $f_{d}$ is an increasing map from ${ }^{D \backslash\{a\}} P$ to $P$, and for all $i<3$, $f_{d}\left(q_{i} \cdot(D \backslash\{a\})\right) \leq q_{i}$. Thus by induction hypothesis, it suffices to prove that $\{d\} \in \mathcal{U}^{f_{d}}$. For all $x, y$ in $P$, we have

$$
\begin{aligned}
f_{d}(x \cdot\{d\} \cup y \cdot(D \backslash\{a, d\})) & =f(x \cdot\{a, d\} \cup y \cdot(D \backslash\{a, d\})) \\
& =x \quad \text { since }\{a, d\} \in \mathcal{U}^{f},
\end{aligned}
$$

which completes the proof of the claim.

- Claim 1.

Now, fix $s \in{ }^{D \backslash\{a, b\}} P$, and let $g_{s}$ be the map defined by

$$
g_{s}: P \times P \rightarrow P, \quad(x, y) \mapsto f(x \cdot\{a\} \cup y \cdot\{b\} \cup s) .
$$

For all $x$ in $P, g_{s}(x, x)=f_{b}(x \cdot\{b\} \cup s)=x$ by claim 1 . Since $g_{s}$ is increasing, it results from lemma 2.4 that $g_{s}$ is a projection.

Claim 2. $(\forall x, y \in P)\left(g_{s}(x, y)=x\right)$.

Proof of claim. Suppose on the contrary that $g_{s}=\pi_{1}^{2}$. Let $y \neq s(c)$ in $P$. Put $t=y \cdot\{b\} \cup s$. Then we have, using claim 1 ,

$$
s(c)=t(c)=f_{c}(t)=f(t \cup t(c) \cdot\{a\})=f(s(c) \cdot\{a\} \cup y \cdot\{b\} \cup s)=g_{s}(s(c), y)=y,
$$

a contradiction.

- Claim 2.

Since the result of claim 2 holds for every $s \in \in^{D \backslash\{a, b\}} P$, we obtain the desired result on $f$. This concludes the study of case 1 .

Case 2. $D$ is arbitrary.

Let $s \in{ }^{D} P$ arbitrary. Let $f_{s}$ be the map defined by

$$
f_{s}:{ }^{P} P \rightarrow P, t \mapsto f\left(\bigcup_{p \in P} t(p) \cdot s^{-1}\{p\}\right)
$$

Then $f_{s}$ is an increasing map from ${ }^{P} P$ to $P$, and for all $i<3, f_{s}\left(q_{i} \cdot P\right)=f\left(q_{i} \cdot D\right) \leq q_{i}$. Therefore, by the result of case 1 , there exists $p_{s} \in P$ such that for all $t \in P P, f_{s}(t)=t\left(p_{s}\right)$. Let $x, y$ in $P$. Then we have

$$
f\left(x \cdot s^{-1}\left\{p_{s}\right\} \cup y \cdot \complement s^{-1}\left\{p_{s}\right\}\right)=f_{s}\left(x \cdot\left\{p_{s}\right\} \cup y \cdot \complement\left\{p_{s}\right\}\right)=x .
$$


Thus $s^{-1}\left\{p_{s}\right\} \in \mathcal{U}^{f}$, and thus $p_{s}=\lim _{\mathcal{U}^{f}} s$.

It follows that

$$
f(s)=f\left(\bigcup_{p \in P} p \cdot s^{-1}\{p\}\right)=f_{s}\left(\bigcup_{p \in P} p \cdot\{p\}\right)=p_{s}=\lim _{\mathcal{U}^{f}} s .
$$

This completes the proof.

3.3. Corollary. Let $f:{ }^{\omega} P \rightarrow P$ be an increasing Borel map such that for all $i<3$, $f\left(q_{i} \cdot \omega\right) \leq q_{i}$. Then $f$ is a projection.

Proof. For all $X \subseteq \omega, X$ belongs to $\mathcal{U}^{f}$ if and only if $p_{0} \cdot X \cup q_{0} \cdot \complement X \in f^{-1}\left\{p_{0}\right\}$. Since $f$ is Borel, $\mathcal{U}^{f}$ is Borel. Thus ([6, theorem 5.7] or [20, theorem 21.4]) $\mathcal{U}^{f}$ is principal, i.e. there exists $n \in \omega$ such that $\mathcal{U}^{f}=(n)$. By theorem 3.2, $f=\pi_{n}^{\omega}$.

Note that the proofs of lemma 3.1 to corollary 3.3 could have been formulated in various more general contexts. Indeed, only lemma 2.4 has been used in the proofs (plus the fact that there are $p$ and $q$ in $P$ such that $p<q$, but if $|P|>1$, then this comes immediately from the fact that every increasing map $f: P \times P \rightarrow P$ such that $f(x, x)=x$ for all $x \in P$ is a projection). Thus the same proofs could have for example given the following result:

3.4. Proposition. Let $Q$ be a partially ordered set such that every increasing map $f: Q \times Q \rightarrow Q$ such that $(\forall x \in Q)(f(x, x) \leq x)$ is a projection. Then for all $n$ in $\omega \backslash\{0\}$, every increasing map $f$ from ${ }^{n} Q$ to $Q$ such that $(\forall x \in Q)(f(x \cdot n) \leq x)$ is a projection. If in addition $Q$ is finite, then every Borel increasing map from ${ }^{\omega} Q$ to $Q$ such that $(\forall x \in Q)(f(x \cdot \omega) \leq x)$ is a projection.

3.5. Corollary. The $\sigma$-bi-frame $\mathbb{E}_{\omega}$ of all Borel lower subsets of ${ }^{\omega} P$ is not 3-variable countably equationally compact.

Proof. For all $m \in \omega$ and all $Q \subseteq P$, put $Q^{m}=\left\{s \in{ }^{\omega} P: s(m) \in Q\right\}$ and $Q^{(m)}=$ $\left\{s \in{ }^{\omega} P:(\forall n \geq m)(s(n) \in Q)\right\}=\bigcap_{n \geq m} Q^{n}$. Consider the following atomic system, with parameters from $\mathbb{E}_{\omega}$ and three unknowns $\mathbf{x}_{0}, \mathbf{x}_{1}, \mathbf{x}_{2}$ :

$$
\left\{\begin{array}{l}
A_{0}^{(m)} \leq \mathbf{x}_{0} \\
A_{1}^{(0)} \leq \mathbf{x}_{1} \\
A_{2}^{(0)} \leq \mathbf{x}_{2} \\
\mathbf{x}_{0} \wedge \mathbf{x}_{1} \wedge \mathbf{x}_{2}=\emptyset \\
\mathbf{x}_{0} \vee \mathbf{x}_{1} \vee \mathbf{x}_{2}={ }^{\omega} P
\end{array}\right.
$$

For all $n \in \omega$, the triple $\left(A_{0}^{n}, A_{1}^{n}, A_{2}^{n}\right)$ is a solution of the finite system

$$
\left\{\begin{array}{l}
A_{0}^{(m)} \leq \mathbf{x}_{0} \\
A_{1}^{(0)} \leq \mathbf{x}_{1} \\
A_{2}^{(0)} \leq \mathbf{x}_{2} \\
\mathbf{x}_{0} \wedge \mathbf{x}_{1} \wedge \mathbf{x}_{2}=\emptyset \\
\mathbf{x}_{0} \vee \mathbf{x}_{1} \vee \mathbf{x}_{2}={ }^{\omega} P
\end{array}\right.
$$


It follows that (3.1) is finitely solvable. We shall prove that (3.1) is not solvable. Otherwise, let $\left(X_{0}, X_{1}, X_{2}\right)$ be a solution of (3.1) in $\mathbb{E}_{\omega}$. By the last two equations on (3.1), there exists a unique map $f:{ }^{\omega} P \rightarrow P$ such that for all $s \in{ }^{\omega} P$ and all $i<3$, we have

$$
f(s) \leq q_{i} \Longleftrightarrow s \in X_{i} .
$$

Since $X_{0}, X_{1}, X_{2}$ are Borel, $f$ is Borel. For all $i<3, q_{i} \cdot \omega$ belongs to $A_{i}^{(0)}$ thus to $X_{i}$, so that $f\left(q_{i} \cdot \omega\right) \leq q_{i}$. Since $X_{0}, X_{1}, X_{2}$ are lower subsets of ${ }^{\omega} P, f$ is increasing. By corollary 3.3 , there exists $n \in \omega$ such that

$$
\left(\forall s \in{ }^{\omega} P\right)(f(s)=s(n)) \text {. }
$$

Let $m=n+1$. We have

$$
p_{0} \cdot m \cup q_{0} \cdot(\omega \backslash m) \in A_{0}^{(m)},
$$

thus, by the first inequality in (3.1),

$$
f\left(p_{0} \cdot m \cup q_{0} \cdot(\omega \backslash m)\right) \leq q_{0} .
$$

But by (3.3), the left-hand side of (3.4) is equal to $p_{0}$, a contradiction.

\section{§. THE CONCLUSION FOR BI-FRAMES.}

In this section, we will still make use of the $P, p_{i}, q_{i}$ of definition $2.2, \pi_{d}^{D}$ of the note after lemma 2.3 and $A_{i}$ of example 2.5. But first, we start with a few topological lemmas.

Lemma 4.1. Let $X$ and $Y$ be Polish spaces and let $f: X \rightarrow Y$ be a continuous, open map. Then the following holds:

(i) For every open dense subset $V$ of $Y, f^{-1}[V]$ is open dense in $X$;

(ii) For every residual subset $F$ of $Y, f^{-1}[F]$ is residual in $X$;

(iii) If in addition $f$ is surjective, then for every residual subset $E$ of $X, f[E]$ is residual in $Y$.

Proof. (i) is easy; (ii) results immediately from (i). Let us prove (iii). Without loss of generality, $E$ is a dense $\mathbf{G}_{\delta}$ subset of $X$. Thus $f[E]$ is an analytical subset of $Y$, thus it has the Baire property, i.e. $f[E]=V \triangle M$ for some open subset $V$ of $Y$ and some meager subset $M$ of $Y$. To prove that $f[E]$ is residual, it suffices to prove that $V$ is dense in $Y$. So let $W$ be a nonempty open subset of $Y$. Since $f$ is surjective and continuous, $f^{-1}[W]$ is a nonempty open subset of $X$. Let $g$ be the restriction of $f$ from $f^{-1}[W]$ to $W$. Then $g$ is continuous and open, thus, by (ii), $g^{-1}[W \backslash M]$ is residual in $f^{-1}[W]$; since $E$ is residual in $X$ and $f^{-1}[W]$ is open in $X$, it follows that $E \cap f^{-1}[W \backslash M]$ is residual in $f^{-1}[W]$, thus nonempty by the Baire category theorem. Thus $(W \backslash M) \cap f[E] \neq \emptyset$, whence $W \cap V \neq \emptyset$, thus proving denseness of $V$. 
4.2. Lemma. Let $m$ in $\omega \backslash\{0\}$. Then the map

$$
\delta_{m}:{ }^{m} \mathcal{P}(\omega) \rightarrow \mathcal{P}(\omega),\left(I_{k}\right)_{k<m} \mapsto \bigcap_{k<m} I_{k}
$$

is surjective, continuous and open.

Proof. The surjectivity is trivial. If $n \in \omega,\left(I_{k}\right)_{k<n}$ and $\left(J_{k}\right)_{k<n}$ are in ${ }^{m} \mathcal{P}(\omega)$ and $I_{k} \cap n=J_{k} \cap n$ for all $k<m$, then $\bigcap_{k<m} I_{k} \cap n=\bigcap_{k<m} J_{k} \cap n$, thus $\delta_{m}$ is continuous. Finally, let $\left(I_{k}\right)_{k<m}$ in ${ }^{m} \mathcal{P}(\omega), J \in \mathcal{P}(\omega)$ and $n \in \omega$ such that $\bigcap_{k<m} I_{k} \cap n=J \cap n$. For all $k<m$, put $J_{k}=\left(I_{k} \cap n\right) \cup(J \backslash n)$. Then $J_{k} \cap n=I_{k} \cap n$ for all $k<m$, and $\bigcap_{k<m} J_{k}=J$. This proves that $\delta_{m}$ is open.

4.3. Lemma. The map

$$
\sigma: \mathcal{P}(\omega) \times \mathcal{P}(\omega) \rightarrow \mathcal{P}(\omega),(I, J) \mapsto I \cup J
$$

is continuous, surjective and open.

Proof. An immediate consequence of lemma 4.2 (using deMorgan's rule).

4.4. Lemma. Let $\mathcal{U}$ be a subset of $\mathcal{P}(\omega)$ satisfying the following conditions.

(i) $\left(\forall^{*} I\right)(I \notin \mathcal{U} \Leftrightarrow \complement I \in \mathcal{U})$;

(ii) $\left(\forall^{*}(I, J)\right)(I \in \mathcal{U} \Rightarrow I \cup J \in \mathcal{U})$;

(iii) $\left(\forall^{*}(I, J)\right)((I \in \mathcal{U}$ and $J \in \mathcal{U}) \Rightarrow I \cap J \in \mathcal{U})$;

(iv) $\mathcal{U}$ has the Baire property.

Then there exists a unique $n \in \omega$ such that we have

$$
\left(\forall^{*} I\right)(I \in \mathcal{U} \Longleftrightarrow n \in I)
$$

Proof. Note that by (i), $\mathcal{U}$ is not meager. Since $\mathcal{U}$ has the Baire property, there exists a meager subset $\mathcal{M}$ of $\mathcal{P}(\omega)$ such that $\mathcal{U} \triangle \mathcal{M}$ is closed (in $\mathcal{P}(\omega)$ ). Let $\mathcal{B}$ be defined by

$$
\mathcal{B}=\{I \in \mathcal{P}(\omega) \backslash \mathcal{M}: I \notin \mathcal{U} \Leftrightarrow \complement I \in \mathcal{U}\}
$$

Then $\mathcal{B}$ is residual and $\mathcal{U} \cap \mathcal{B}$ is closed in $\mathcal{B}$. Replacing $\mathcal{U}$ by $\mathcal{U} \cap \mathcal{B}$, we see that without loss of generality, one may assume that $\mathcal{U}$ is a subset of $\mathcal{B}$, closed for the relative topology; furthermore, $\mathcal{B}$ is closed under complementation and we have

(i') $(\forall I \in \mathcal{B})(I \notin \mathcal{U} \Leftrightarrow \complement I \in \mathcal{U})$.

By (ii) and (iii), there exists a residual subset $\mathcal{C}$ of $\mathcal{B} \times \mathcal{B}$ such that the following holds:

(a) $(\forall(I, J) \in \mathcal{C})(I \in \mathcal{U} \Rightarrow I \cup J \in \mathcal{U})$;

(b) $(\forall(I, J) \in \mathcal{C})((I \in \mathcal{U}$ and $J \in \mathcal{U}) \Rightarrow I \cap J \in \mathcal{U})$. 
Define inductively $\mathcal{C}_{n} \subseteq{ }^{n} \mathcal{B}$ (all $n \in \omega \backslash\{0\}$ ) as follows. Put $\mathcal{C}_{1}=\mathcal{B}$ and for all $n \in \omega \backslash\{0\}$,

$$
\mathcal{C}_{n+1}=\left\{\left(I_{k}\right)_{k \leq n} \in{ }^{n+1} \mathcal{B}:\left(I_{k}\right)_{k<n} \in \mathcal{C}_{n} \text { and }\left(\bigcap_{k<n} I_{k}, I_{n}\right) \in \mathcal{C}\right\}
$$

Claim. For all $n \in \omega \backslash\{0\}, \mathcal{C}_{n}$ is a residual subset of ${ }^{n} \mathcal{B}$ and $\delta_{n}\left[\mathcal{C}_{n} \cap{ }^{n} \mathcal{U}\right] \subseteq \mathcal{U}$.

Proof of claim. By lemmas 4.1 and 4.2 , it is easy to verify by induction that for all $n \in \omega \backslash\{0\}, \mathcal{C}_{n}$ is residual in ${ }^{n} \mathcal{B}$. Then, using (b) above, it is immediate to verify by induction that for all $n \in \omega \backslash\{0\}, \mathcal{C}_{n}$ has the required property.

- Claim

Suppose now that the following condition holds:

$$
(\forall m \in \omega)\left(\exists^{*} I\right)(I \in \mathcal{U} \text { and } I \cap m=\emptyset) .
$$

We prove that $\mathcal{U}$ is dense in $\mathcal{B}$. Let $m \in \omega$ and let $s \subseteq m$. By assumption, the set $\mathcal{V}=\{I \in \mathcal{U}: I \cap m=\emptyset\}$ is not meager. Since $\mathcal{B}$ is residual, $\mathcal{W}=\{J \in \mathcal{B}: J \cap m=s\}$ is not meager. Since $\mathcal{C}$ is residual, it results from the Kuratowski-Ulam theorem that $\mathcal{C} \cap(\mathcal{V} \times \mathcal{W}) \neq \emptyset$. Let $(I, J) \in \mathcal{C} \cap(\mathcal{V} \times \mathcal{W})$. Then $I \in \mathcal{U}, I \cap m=\emptyset, J \in \mathcal{B}, J \cap m=s$, thus $(I \cup J) \cap m=s$; furthermore, $I \cup J \in \mathcal{U}$ by (a). This proves the denseness of $\mathcal{U}$ in $\mathcal{P}(\omega)$. Since $\mathcal{U}$ is closed in $\mathcal{B}$, we obtain $\mathcal{U}=\mathcal{B}$, which contradicts (i').

Hence we have proved that (4.1) is not possible. Thus there exists $m$ in $\omega$ such that

$$
\left(\forall^{*} I\right)(I \in \mathcal{U} \Rightarrow I \cap m \neq \emptyset) .
$$

Suppose that the following holds:

$$
(\forall k<m)\left(\exists^{*} I\right)(I \in \mathcal{U} \text { and } k \notin I) .
$$

Thus for all $k<m$, there exists a nonempty open subset $\mathcal{V}_{k}$ of $\mathcal{P}(\omega)$ such that $\mathcal{V}_{k}^{\prime}=$ $\left\{I \in \mathcal{V}_{k}: I \in \mathcal{U}\right.$ and $\left.k \notin I\right\}$ is residual in $\mathcal{V}_{k}$. By lemmas 4.1 and $4.2, \mathcal{V}=\delta_{m}\left[\prod_{k<m} \mathcal{V}_{k}\right]$ is open (nonempty) and $\mathcal{V}^{\prime}=\delta_{m}\left[\mathcal{C}_{m} \cap \prod_{k<m} \mathcal{V}_{k}^{\prime}\right]$ is residual in $\mathcal{V}$. By (4.2), there exists $I \in \mathcal{V}^{\prime}$ satisfying $I \in \mathcal{U} \Rightarrow I \cap m \neq \emptyset$. But $I=\bigcap_{k<m} I_{k}$ where $\left(I_{k}\right)_{k<m} \in \mathcal{C}_{m}$ and for all $k<m, I_{k} \in \mathcal{V}_{k}^{\prime} \subseteq \mathcal{U}$. By the claim, it follows that $I \in \mathcal{U}$, so that $I \cap m \neq \emptyset$. On the other hand, $k \notin I_{k}$ for all $k<m$, thus $I \cap m=\emptyset$, a contradiction.

Hence we have proved that (4.3) is not possible. Thus there exists $n<m$ such that $\left(\forall^{*} I\right)(I \in \mathcal{U} \Rightarrow n \in I)$. Using (i'), it is then easy to prove that in fact, we have $\left(\forall^{*} I\right)(I \in \mathcal{U} \Leftrightarrow n \in I)$. If $n^{\prime}$ is another integer with that property, then we have $\left(\forall^{*} I\right)\left(n \in I \Leftrightarrow n^{\prime} \in I\right)$, whence $n=n^{\prime}$ by the Baire category theorem. 
Now, we are going to construct the final example.

Equip $\mathcal{P}(P)$ with the preordering $\leq$ defined by

$$
X \leq Y \Longleftrightarrow[(\forall x \in X)(\exists y \in Y)(x \leq y) \text { and }(\forall y \in Y)(\exists x \in X)(x \leq y)]
$$

Put $\mathcal{P}^{*}(P)=\mathcal{P}(P) \backslash\{\emptyset\}$.

Let $\mathcal{D}$ be the set of all decreasing (for the inclusion) sequences of elements of $\mathcal{P}^{*}(P)$. It is important to note that since $P$ is finite, $\mathcal{D}$ is countable. Equip $\mathcal{D}$ with the discrete topology and with the componentwise preordering $(X \leq Y$ if and only if for all $n \in \omega$, $X(n) \leq Y(n)$ where $\leq$ is the preordering defined in (4.4)).

Let $\Omega$ be the subset of ${ }^{\omega} P \times \mathcal{D}$ defined by

$$
\Omega=\left\{(x, X) \in{ }^{\omega} P \times \mathcal{D}:(\forall n \in \omega)(x(n) \in X(n))\right\},
$$

endowed with the topology induced by the product topology. Thus $\Omega$ is a closed subset of a Polish space, thus $\Omega$ is a Polish space. Equip $\Omega$ with the componentwise preordering, still denoted by $\leq$. For all $X$ in $\mathcal{D}$, put $\Omega_{X}=\left\{x \in{ }^{\omega} P:(x, X) \in \Omega\right\}=\prod_{n \in \omega} X(n)$, so that $\Omega_{X}$ is a closed subset of ${ }^{\omega} P$.

4.5. Lemma. Let $U$ be a lower subset of $\Omega$. Then $\operatorname{Cl}(U)$ is a lower subset of $\Omega$.

Proof. Let $(y, Y) \in \mathrm{Cl}(U)$ and let $(x, X) \leq(y, Y)$. We prove that $(x, X) \in \mathrm{Cl}(U)$. Let $m \in \omega$. There exists $y^{\prime} \in \Omega_{Y}$ such that $y^{\prime} \uparrow_{m}=y \Upsilon_{m}$. For all $n \geq m, y^{\prime}(n) \in Y(n)$ and $X(n) \leq Y(n)$, thus (see (4.4)) there exists $z(n) \in X(n)$ such that $z(n) \leq y^{\prime}(n)$. Let $x^{\prime}=x \uparrow_{m} \cup z$. It is easy to verify that by construction, $\left(x^{\prime}, X\right) \in \Omega$ and that $x^{\prime} \leq y^{\prime}$. But $\left(y^{\prime}, Y\right) \in U$ and $U$ is a lower set, thus $\left(x^{\prime}, X\right) \in U$. Since $x^{\prime} \uparrow_{m}=x \uparrow_{m}$, we have proved that $(x, X) \in \mathrm{Cl}(U)$.

4.6. Lemma. Let $U$ be a lower subset of $\Omega$. Then $\operatorname{Int}(U)$ is a lower subset of $\Omega$.

Proof. Let $(y, Y) \in \operatorname{Int}(U)$ and let $(x, X) \leq(y, Y)$. We prove that $(x, X) \in \operatorname{Int}(U)$. By definition, there exists $m$ in $\omega$ such that

$$
\left(\forall y^{\prime} \in \Omega_{Y}\right)\left(y^{\prime} \uparrow_{m}=y \uparrow_{m} \Rightarrow\left(y^{\prime}, Y\right) \in U\right)
$$

We shall prove that the following holds:

$$
\left(\forall x^{\prime} \in \Omega_{X}\right)\left(x^{\prime} \uparrow_{m}=x \uparrow_{m} \Rightarrow\left(x^{\prime}, X\right) \in U\right)
$$

So, let $x^{\prime} \in \Omega_{X}$ such that $x^{\prime} \uparrow_{m}=x \uparrow_{m}$. For all $n \geq m, x^{\prime}(n) \in X(n)$ and $X(n) \leq Y(n)$, thus (see (4.4)) there exists $z(n) \in Y(n)$ such that $x^{\prime}(n) \leq z(n)$. Let $y^{\prime}=y \Upsilon_{m} \cup z$. It is easy to verify that by construction, $\left(y^{\prime}, Y\right) \in \Omega$ and that $x^{\prime} \leq y^{\prime}$. By $(4.5),\left(y^{\prime}, Y\right) \in U$; since $U$ is a lower set, $\left(x^{\prime}, X\right) \in U$. Thus we have verified (4.6), which implies that $(x, X) \in \operatorname{Int}(U)$. 
Now, let $\mathbb{B}$ the Boolean algebra of regular open subsets of $\Omega$. It is well-known that $\mathbb{B}$ is a complete Boolean algebra.

4.7. Lemma. The set $\mathbb{E}$ of all regular open lower subsets of $\Omega$ is a sublattice of $\mathbb{B}$, closed under the operations of (infinite) meet and join. Thus it is a sub-bi-frame of $\mathbb{B}$.

Proof. Let $\left(U_{i}\right)_{i \in I}$ be an arbitrary family of regular open lower subsets of $\Omega$. Then the meet and the join of this family in $\mathbb{B}$ are respectively given by

$$
\begin{gathered}
\bigwedge_{i \in I} U_{i}=\operatorname{Int} \mathrm{Cl}\left(\bigcap_{i \in I} U_{i}\right)=\operatorname{Int}\left(\bigcap_{i \in I} U_{i}\right), \\
\bigvee_{i \in I} U_{i}=\operatorname{Int} \mathrm{Cl}\left(\bigcup_{i \in I} U_{i}\right) .
\end{gathered}
$$

Using lemmas 4.5 and 4.6 , one sees easily that $\bigwedge_{i \in I} U_{i}$ and $\bigvee_{i \in I} U_{i}$ are lower subsets of $\Omega$.

It is this $\mathbb{E}$ that is our candidate for failure of 3-variable countable equational compactness for bi-frames.

For every $Q \subseteq P$ and every $m \in \omega$, define subsets $Q^{(m)}$ and $Q^{m}$ of $\Omega$ by putting

$$
\left\{\begin{array}{l}
Q^{(m)}=\{(x, X) \in \Omega: X(m) \subseteq Q\} \\
Q^{m}=\{(x, X) \in \Omega: x(m) \in Q\}
\end{array}\right.
$$

(Warning: these notations do not have the same meaning as in the proof of corollary 3.5). Note that $\left(Q^{(m)}\right)_{m \in \omega}$ is increasing for the inclusion.

4.8. Lemma. For every $m \in \omega$ and every $Q \subseteq P, Q^{(m)} \subseteq Q^{m}$ and $Q^{(m)}$ and $Q^{m}$ are clopen subsets of $\Omega$.

Proof. Easy.

Note that using the fact that the $A_{i}^{m}$ 's are clopen, it is easy to verify that for all $m \in \omega$, the following holds:

$$
\left\{\begin{array}{l}
A_{0}^{m} \wedge A_{1}^{m} \wedge A_{2}^{m}=A_{0}^{m} \cap A_{1}^{m} \cap A_{2}^{m}=\emptyset \\
A_{0}^{m} \vee A_{1}^{m} \vee A_{2}^{m}=A_{0}^{m} \cup A_{1}^{m} \cup A_{2}^{m}=\Omega
\end{array}\right.
$$

Thus we obtain immediately the following

4.9. Lemma. The following atomic system with parameters from $\mathbb{E}$ and three unknowns $\mathbf{x}_{0}, \mathbf{x}_{1}$ and $\mathbf{x}_{2}$ :

$$
\left\{\begin{array}{l}
A_{0}^{(m)} \leq \mathbf{x}_{0} \quad(\text { all } m \text { in } \omega) \\
A_{1}^{(0)} \leq \mathbf{x}_{1} \\
A_{2}^{(0)} \leq \mathbf{x}_{2} \\
\mathbf{x}_{0} \wedge \mathbf{x}_{1} \wedge \mathbf{x}_{2}=\emptyset \\
\mathbf{x}_{0} \vee \mathbf{x}_{1} \vee \mathbf{x}_{2}=\Omega
\end{array}\right.
$$

is finitely solvable in $\mathbb{E}$. 
Note that the parameters of this system lie in $\mathbb{E}$ (they are in fact clopen).

However, we shall prove that (4.8) is not solvable in $\mathbb{E}$. From now on until the end of this section, suppose that $\left(\boldsymbol{X}_{0}, \boldsymbol{X}_{1}, \boldsymbol{X}_{2}\right)$ is a solution of (4.8) in $\mathbb{E}$. Thus $\boldsymbol{X}_{0}, \boldsymbol{X}_{1}$ and $\boldsymbol{X}_{2}$ are regular open lower subsets of $\Omega$ and the following holds:

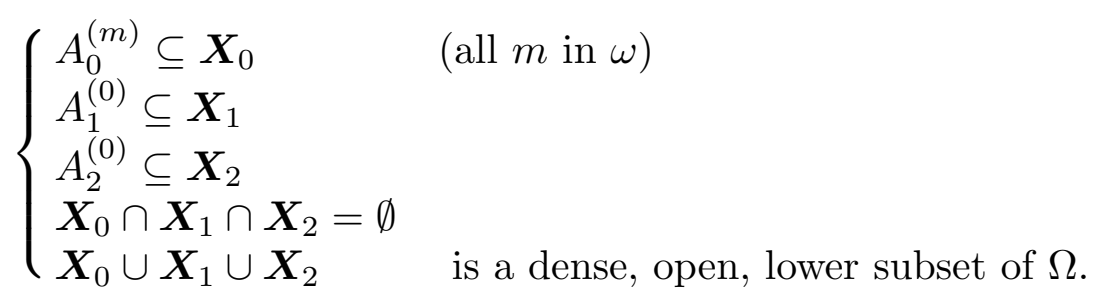

Put $\Omega^{*}=\boldsymbol{X}_{0} \cup \boldsymbol{X}_{1} \cup \boldsymbol{X}_{2}$, so that $\Omega^{*}$ is a dense, open lower subset of $\Omega$; for all $X \in \mathcal{D}$, put $\Omega_{X}^{*}=\left\{x \in{ }^{\omega} P:(x, X) \in \Omega^{*}\right\}$, so that $\Omega_{X}^{*}$ is open dense in $\Omega_{X}$. Since $\boldsymbol{X}_{0} \cap \boldsymbol{X}_{1} \cap \boldsymbol{X}_{2}=\emptyset$, there exists a unique map $f: \Omega^{*} \rightarrow P$ such that for all $\boldsymbol{x} \in \Omega^{*}$ and all $i<3$, we have

$$
f(\boldsymbol{x}) \leq q_{i} \Longleftrightarrow \boldsymbol{x} \in \boldsymbol{X}_{i}
$$

Since $\boldsymbol{X}_{0}, \boldsymbol{X}_{1}$ and $\boldsymbol{X}_{2}$ are lower sets, $f$ is increasing. Furthermore, for all $i<3$, if $3=\{i, j, k\}$, then we have $f^{-1}\left\{p_{i}\right\}=\boldsymbol{X}_{j} \cap \boldsymbol{X}_{k}$ and $f^{-1}\left\{q_{i}\right\}=\boldsymbol{X}_{i} \backslash\left(\boldsymbol{X}_{j} \cup \boldsymbol{X}_{k}\right)$, thus $f$ is a Borel map (and even a Baire class 1 map).

For all $n \in \omega \backslash\{0\}$ and all $a_{1}, \ldots, a_{n}$ in $P$, denote by $\left[a_{1}, \ldots, a_{n}\right]$ the element $X$ of $\mathcal{D}$ defined by $X(k)=\left\{a_{1}, \ldots, a_{n}\right\}$ for all $k \in \omega$.

4.10. Lemma. $\mathcal{B}=\left\{I \subseteq \omega:(\forall p, q \in P)\left((p \cdot I \cup q \cdot \complement I,[p, q]) \in \Omega^{*}\right)\right\}$ is an open dense subset of $\mathcal{P}(\omega)$.

Proof. Since $P$ is finite, it suffices to prove that for all $p, q$ in $P$, the set $\mathcal{B}_{p q}=\{I \subseteq \omega$ : $\left.(p \cdot I \cup q \cdot \complement I,[p, q]) \in \Omega^{*}\right\}$ is open dense in $\mathcal{P}(\omega)$. We distinguish two cases.

Case 1. $p=q$.

Then $\Omega_{[p, q]}$ is the singleton $\{\langle p, p, \ldots\rangle\}$, thus $\Omega_{[p, q]}^{*}=\Omega_{[p, q]}$ and $\mathcal{B}_{p q}=\mathcal{P}(\omega)$.

Case 2. $p \neq q$.

Then $\Omega_{[p, q]}={ }^{\omega}\{p, q\}$. Since the map $\mathcal{P}(\omega) \rightarrow{ }^{\omega}\{p, q\}, I \mapsto p \cdot I \cup q \cdot$ C $I$ is a homeomorphism, the conclusion follows from the fact that $\left\{x \in{ }^{\omega}\{p, q\}:(x,[p, q]) \in \Omega^{*}\right\}=\Omega_{[p, q]}^{*}$ is open dense in $\Omega_{[p, q]}$.

For all $I$ in $\mathcal{B}$, let $f_{I}$ be the map defined (by definition of $\mathcal{B}$ ) by

$$
f_{I}: P \times P \rightarrow P,(p, q) \mapsto f(p \cdot I \cup q \cdot \complement I,[p, q])
$$

It is clear that $f_{I}$ is an increasing map from $P \times P$ to $P$. Furthermore, for all $i<3$, $\left(q_{i} \cdot \omega,\left[q_{i}\right]\right) \in A_{i}^{(0)} \subseteq \boldsymbol{X}_{i}$, whence $f\left(q_{i} \cdot \omega,\left[q_{i}\right]\right) \leq q_{i}$, i.e. $f_{I}\left(q_{i}, q_{i}\right) \leq q_{i}$. By lemma $2.4, f_{I}$ is a projection, i.e. either $f_{I}=\pi_{0}^{2}$ or $f_{I}=\pi_{1}^{2}$. 
Let $\mathcal{U}=\left\{I \in \mathcal{B}: f_{I}=\pi_{0}^{2}\right\}$. It is clear that $\mathcal{U}$ has the Baire property (it is even open).

4.11. Lemma. The following holds:

(i) $(\forall I \in \mathcal{B})(I \notin \mathcal{U} \Leftrightarrow \complement I \in \mathcal{U})$;

(ii) $(\forall I, J \in \mathcal{B})((I \in \mathcal{U}$ and $I \subseteq J) \Rightarrow J \in \mathcal{U})$;

(iii) $\left(\forall^{*}(I, J) \in \mathcal{B} \times \mathcal{B}\right)(I \in \mathcal{U} \Rightarrow I \cup J \in \mathcal{U})$.

Proof. (i) comes from the fact that $f_{I}$ is either $\pi_{0}^{2}$ or $\pi_{1}^{2}$.

(ii) Let $I$ in $\mathcal{U}$ and $J$ in $\mathcal{B}$ such that $I \subseteq J$. Suppose $J \notin \mathcal{U}$. Let $p$ and $q$ in $P$ such that $p<q$, for example $p=p_{0}$ and $q=q_{1}$. Since $I \subseteq J$ and $p \leq q$ and $f$ is increasing, we have

$$
f(p \cdot J \cup q \cdot \complement J,[p, q]) \leq f(p \cdot I \cup q \cdot \complement I,[p, q]) .
$$

But since $J \notin \mathcal{U}$, the left-hand side of the inequality above is $q$, while since $I \in \mathcal{U}$, the right-hand side of the inequality above is $p$. Since $p<q$, we obtain a contradiction.

(iii) Let $\mathcal{C}=\{(I, J) \in \mathcal{B} \times \mathcal{B}: I \cup J \in \mathcal{B}\}$. Since $\mathcal{B}$ is a dense open subset of $\mathcal{P}(\omega)$ and by lemmas 4.1 and $4.3, \mathcal{C}$ is a dense open subset of $\mathcal{P}(\omega) \times \mathcal{P}(\omega)$. Let $(I, J) \in \mathcal{C}$ such that $I \in \mathcal{U}$. Then $I \in \mathcal{U}, I \cup J \in \mathcal{B}$ and $I \subseteq I \cup J$, thus $I \cup J \in \mathcal{U}$ by (ii).

For all $n$ in $\omega \backslash\{0\}$, let $\Pi_{n}(\omega)$ be defined by

$$
\Pi_{n}(\omega)=\left\{\left(I_{k}\right)_{k<n} \in{ }^{n} \mathcal{P}(\omega): \bigwedge_{k<l<n}\left(I_{k} \cap I_{l}=\emptyset\right) \text { and } \bigcup_{k<n} I_{k}=\omega\right\}
$$

Thus $\Pi_{n}(\omega)$ is a closed subset of ${ }^{n} \mathcal{P}(\omega)$.

4.12. Lemma. For all $n$ in $\omega \backslash\{0\}$ and all $a_{k}(k<n)$ in $P$, the map

$$
\pi: \Pi_{n}(\omega) \rightarrow{ }^{\omega}\left\{a_{k}: k<n\right\},\left(I_{k}\right)_{k<n} \mapsto \bigcup_{k<n} a_{k} \cdot I_{k}
$$

is continuous, open and surjective.

Proof. It is trivial that $\pi$ is surjective. If $\left(I_{k}\right)_{k<n}$ and $\left(J_{k}\right)_{k<n}$ are two elements of $\Pi_{n}(\omega)$ and $m \in \omega$ such that $(\forall k<n)\left(I_{k} \cap m=J_{k} \cap m\right)$, then $\pi\left(\left(I_{k}\right)_{k<n}\right) \uparrow_{m}=\pi\left(\left(J_{k}\right){ }_{k<n}\right) \uparrow_{m}$; thus $\pi$ is continuous. If $\left(I_{k}\right)_{k<n} \in \Pi_{n}(\omega), x=\pi\left(\left(I_{k}\right)_{k<n}\right), m \in \omega$ and $y \in{ }^{\omega}\left\{a_{k}: k<n\right\}$ are such that $x \Upsilon_{m}=y \Upsilon_{m}$, put $J_{k}=\left(I_{k} \cap m\right) \cup\left(y^{-1}\left\{a_{k}\right\} \backslash m\right)$ for all $k<n$. Then for all $k<n, I_{k} \cap m=J_{k} \cap m$ and $\pi\left(\left(J_{k}\right)_{k<n}\right)=y$. Thus $\pi$ is open.

4.13. Lemma. Both following maps:

$$
\begin{gathered}
\mathcal{P}(\omega) \times \mathcal{P}(\omega) \rightarrow \Pi_{4}(\omega),(I, J) \mapsto\langle I \cap J, I \backslash J, J \backslash I, \complement I \cap \complement J\rangle, \\
\Pi_{4}(\omega) \rightarrow \mathcal{P}(\omega) \times \mathcal{P}(\omega),\left\langle I_{0}, I_{1}, I_{2}, I_{3}\right\rangle \mapsto\left(I_{0} \cup I_{1}, I_{0} \cup I_{2}\right)
\end{gathered}
$$

are homeomorphisms, inverse from each other.

Proof. Easy. 
4.14. Lemma. The following set

$$
\begin{aligned}
& \mathcal{B}^{\prime}=\{(I, J) \in \mathcal{B} \times \mathcal{B}:\left(\forall a_{0}, a_{1}, a_{2}, a_{3} \in P\right) \\
&\left.\left(\left(a_{0} \cdot(I \cap J) \cup a_{1} \cdot(I \backslash J) \cup a_{2} \cdot(J \backslash I) \cup a_{3} \cdot(\complement I \cap \complement J),\left[a_{0}, a_{1}, a_{2}, a_{3}\right]\right) \in \Omega^{*}\right)\right\}
\end{aligned}
$$

is an open dense subset of $\mathcal{P}(\omega) \times \mathcal{P}(\omega)$.

Proof. Since $\mathcal{B}$ is open dense in $\mathcal{P}(\omega)$ (lemma 4.10), it suffices to prove that for all $\vec{a}=\left\langle a_{0}, a_{1}, a_{2}, a_{3}\right\rangle$ in ${ }^{4} P$, the set

$\mathcal{B}_{\vec{a}}=\left\{(I, J) \in \mathcal{B} \times \mathcal{B}:\left(a_{0} \cdot(I \cap J) \cup a_{1} \cdot(I \backslash J) \cup a_{2} \cdot(J \backslash I) \cup a_{3} \cdot(\complement I \cap \complement J),\left[a_{0}, a_{1}, a_{2}, a_{3}\right]\right) \in \Omega^{*}\right\}$

is open dense in $\mathcal{P}(\omega) \times \mathcal{P}(\omega)$. But this is an immediate consequence of lemmas $4.1,4.12$ and 4.13, together with the fact that $\Omega_{\left[a_{0}, a_{1}, a_{2}, a_{3}\right]}^{*}$ is dense in $\Omega_{\left[a_{0}, a_{1}, a_{2}, a_{3}\right]}$.

4.15. Lemma. $\left(\forall^{*}(I, J)\right)[(I \in \mathcal{U}$ and $J \in \mathcal{U}) \Rightarrow I \cap J \in \mathcal{U}]$.

Proof. By lemma 4.14, it suffices to prove that for all $(I, J) \in \mathcal{B}^{\prime}$ such that $I \in \mathcal{U}$ and $J \in \mathcal{U}$, we have $I \cap J \in \mathcal{U}$. Since $(I, J) \in \mathcal{B}^{\prime}$, the function $g$ defined by

$g:{ }^{4} P \rightarrow P,\left\langle a_{0}, a_{1}, a_{2}, a_{3}\right\rangle \mapsto f\left(a_{0} \cdot(I \cap J) \cup a_{1} \cdot(I \backslash J) \cup a_{2} \cdot(J \backslash I) \cup a_{3} \cdot(\complement I \cap \complement J),\left[a_{0}, a_{1}, a_{2}, a_{3}\right]\right)$

is defined everywhere on ${ }^{4} P$. It is clearly increasing. Furthermore, for all $i<3,\left[q_{i}\right] \in A_{i}$, thus, by (4.9), $g\left(q_{i}, q_{i}, q_{i}, q_{i}\right) \leq q_{i}$. By theorem 3.2 (finite case), $g$ is a projection, i.e. $g=\pi_{k}^{4}$ for some $k<4$. We shall prove that $k=0$, by distinguishing cases.

Case 1. $k=1$ or $k=3$.

Then for all $p, q$ in $P$, we have $g(p, q, p, q)=q$. However, $g(p, q, p, q)=f(p \cdot J \cup q$. $\complement J,[p, q])=p$ since $J \in \mathcal{U}$, a contradiction.

Case 2. $k=2$.

Then for all $p, q$ in $P$, we have $g(p, p, q, q)=q$. However, $g(p, p, q, q)=f(p \cdot I \cup q$. $\complement I,[p, q])=p$ since $I \in \mathcal{U}$, a contradiction.

Therefore, we have proved that $k=0$. It follows that for all $p, q$ in $P$, we have $f(p \cdot(I \cap J) \cup q \cdot \complement(I \cap J))=g(p, q, q, q)=p$, whence $I \cap J \in \mathcal{U}$.

4.16. Lemma. There exists $n$ in $\omega$ such that $\left(\forall^{*} I\right)(I \in \mathcal{U} \Leftrightarrow n \in I)$.

Proof. An immediate consequence of lemma 4.11 (i,iii), lemma 4.15 and lemma 4.4.

From now on until theorem 4.19 fix the integer $n$ of lemma 4.16 , and put $m=n+1$. Let $\mathcal{B}^{*}$ be a residual subset of $\mathcal{B}$, closed under complementation, such that for all $I$ in $\mathcal{B}^{*}$, $I \in \mathcal{U}$ if and only if $n \in I$. For all $p, q$ and $r$ in $P$, define $X(p, q, r) \in \mathcal{D}$ by

$$
(\forall k \in \omega) X(p, q, r)(k)= \begin{cases}\{p, q, r\} & (\text { for } k<m) \\ \{q, r\} & (\text { for } k \geq m)\end{cases}
$$


4.17. Lemma. The following set:

$$
\mathcal{B}^{\prime \prime}=\left\{I \subseteq \omega \backslash m:(\forall p, q, r \in P)\left((p \cdot m \cup q \cdot I \cup r \cdot(\omega \backslash m \backslash I), X(p, q, r)) \in \Omega^{*}\right)\right\}
$$

is a dense open subset of $\mathcal{P}(\omega \backslash m)$.

Proof. It suffices to prove that for all $p, q, r$ in $P$, the following set

$$
\mathcal{B}_{p q r}=\left\{I \subseteq \omega \backslash m:(p \cdot m \cup q \cdot I \cup r \cdot(\omega \backslash m \backslash I), X(p, q, r)) \in \Omega^{*}\right\}
$$

is open dense in $\mathcal{P}(\omega \backslash m)$. We argue by cases.

Case 1. $p \in\{q, r\}$.

Thus $X(p, q, r)=[q, r]$. If $p=q$, it suffices to prove that the set

$$
\left\{I \subseteq \omega \backslash m:(q \cdot(m \cup I) \cup r \cdot(\omega \backslash(m \cup I)),[q, r]) \in \Omega^{*}\right\}
$$

is open dense in $\mathcal{P}(\omega \backslash m)$. But this is an immediate consequence of lemma 4.10. If $p=r$, it suffices to prove that the set

$$
\left\{I \subseteq \omega \backslash m:(q \cdot I \cup r \cdot(\omega \backslash I),[q, r]) \in \Omega^{*}\right\}
$$

is open dense in $\mathcal{P}(\omega \backslash m)$. But this is again an immediate consequence of lemma 4.10.

Case 2. $p \notin\{q, r\}$ and $q=r$.

Then $X(p, q, r)(k)=\{q\}$ for all $k \geq m$, whence $\Omega_{X(p, q, r)}$ is finite (of size $2^{m}$ ), thus discrete. Thus $\Omega_{X(p, q, r)}^{*}=\Omega_{X(p, q, r)}$, i.e. for all $x \in \Omega_{X(p, q, r)},(x, X(p, q, r)) \in \Omega^{*}$. This holds in particular for $x=p \cdot m \cup q \cdot(\omega \backslash m)$.

Case 3. $p \neq q, p \neq r$ and $q \neq r$.

Then $\left\{x \in{ }^{\omega \backslash m}\{q, r\}: p \cdot m \cup x \in \Omega_{X(p, q, r)}^{*}\right\}$ is open dense in ${ }^{\omega \backslash m}\{q, r\}$. Since $q \neq r$, the map $\mathcal{P}(\omega \backslash m) \rightarrow{ }^{\omega \backslash m}\{q, r\}, I \mapsto q \cdot I \cup r \cdot(\omega \backslash m \backslash I)$ is a homeomorphism. The conclusion follows.

Now for all $I$ in $\mathcal{B}^{\prime \prime}$, let $g_{I}$ be the map defined by

$$
g_{I}:{ }^{3} P \rightarrow P,\langle p, q, r\rangle \mapsto f(p \cdot m \cup q \cdot I \cup r \cdot(\omega \backslash m \backslash I), X(p, q, r)) .
$$

It is clear that $g_{I}$ is increasing. For all $i<3, X\left(q_{i}, q_{i}, q_{i}\right)(0) \subseteq A_{i}$, thus $g_{I}\left(q_{i}, q_{i}, q_{i}\right) \leq$ $q_{i}$. By lemma 3.1, there exists $l<3$ such that $g=\pi_{l}^{3}$. We shall prove that $l=0$ (for all $\left.I \in \mathcal{B}^{\prime \prime}\right)$.

Thus define $\mathcal{V}$ and $\mathcal{W}$ by

$$
\begin{gathered}
\mathcal{V}=\left\{I \subseteq \omega \backslash m: g_{I}=\pi_{1}^{3}\right\} \\
\mathcal{W}=\left\{I \subseteq \omega \backslash m: g_{I}=\pi_{2}^{3}\right\}
\end{gathered}
$$


Thus we also have

$$
\begin{gathered}
\mathcal{V}=\left\{I \in \mathcal{B}^{\prime \prime}:\left(p_{0} \cdot m \cup p_{1} \cdot I \cup p_{2} \cdot(\omega \backslash m \backslash I), X\left(p_{0}, p_{1}, p_{2}\right)\right) \in \boldsymbol{X}_{0} \cap \boldsymbol{X}_{2}\right\}, \\
\mathcal{W}=\left\{I \in \mathcal{B}^{\prime \prime}:\left(p_{0} \cdot m \cup p_{1} \cdot I \cup p_{2} \cdot(\omega \backslash m \backslash I), X\left(p_{0}, p_{1}, p_{2}\right)\right) \in \boldsymbol{X}_{0} \cap \boldsymbol{X}_{1}\right\},
\end{gathered}
$$

whence $\mathcal{V}$ and $\mathcal{W}$ are open subsets of $\mathcal{P}(\omega)$.

4.18. Lemma. $\mathcal{V}=\mathcal{W}=\emptyset$.

Proof. Evaluating $g_{I}(r, q, r)$ yields the following statement:

$$
(\forall I \in \mathcal{V})(\forall q, r \in P)(f(q \cdot I \cup r \cdot(\omega \backslash I),[q, r])=q)
$$

If $\mathcal{V} \neq \emptyset$, then, since $\mathcal{V}$ is open and $\mathcal{B}^{*}$ is residual, $\mathcal{V} \cap \mathcal{B}^{*} \neq \emptyset$. Let $I \in \mathcal{V} \cap \mathcal{B}^{*}$. Since $I \subseteq \omega \backslash m$, we have $n \notin I$. Since $I \in \mathcal{B}^{*}$, it follows that $\complement I \in \mathcal{U}$, thus for all $q, r$ in $P$, $f(q \cdot I \cup r \cdot(\omega \backslash I),[q, r])=r$. But this contradicts (4.10). Therefore, $\mathcal{V}=\emptyset$.

Similarly, evaluating $g_{I}(q, q, r)$ yields the following statement:

$$
(\forall I \in \mathcal{W})(\forall q, r \in P)(f(q \cdot(m \cup I) \cup r \cdot(\omega \backslash m \backslash I),[q, r])=r)
$$

If $\mathcal{W} \neq \emptyset$, then $\mathcal{W}^{\prime}=\{m \cup I: I \in \mathcal{W}\}$ is a nonempty open subset of $\mathcal{P}(\omega)$, thus, since $\mathcal{B}^{*}$ is residual, $\mathcal{W}^{\prime} \cap \mathcal{B}^{*} \neq \emptyset$. Let $I \in \mathcal{W}$ such that $m \cup I \in \mathcal{B}^{*}$. Since $n \in m \cup I$ and $m \cup I \in \mathcal{B}^{*}$, we have $m \cup I \in \mathcal{U}$, whence for all $q, r$ in $P, f(q \cdot(m \cup I) \cup r \cdot(\omega \backslash m \backslash I),[q, r])=q$. But this contradicts (4.11). Therefore, $\mathcal{W}=\emptyset$.

Now we can conclude! Indeed, by lemma 4.18, the following holds:

$$
\left(\forall I \in \mathcal{B}^{\prime \prime}\right)(\forall p, q, r \in P)[f(p \cdot m \cup q \cdot I \cup r \cdot(\omega \backslash m \backslash I), X(p, q, r))=p]
$$

Taking $p=p_{0}$ and $q=r=q_{0}$ and observing that $\mathcal{B}^{\prime \prime} \neq \emptyset$, we obtain that

$$
f\left(p_{0} \cdot m \cup q_{0} \cdot(\omega \backslash m), X\left(p_{0}, q_{0}, q_{0}\right)\right)=p_{0} .
$$

On the other hand, $X\left(p_{0}, q_{0}, q_{0}\right)(m)=\left\{q_{0}\right\} \subseteq A_{0}$, thus

$$
\left(p_{0} \cdot m \cup q_{0} \cdot(\omega \backslash m), X\left(p_{0}, q_{0}, q_{0}\right)\right) \in A_{0}^{(m)} .
$$

It follows from (4.9) that $f\left(p_{0} \cdot m \cup q_{0} \cdot(\omega \backslash m), X\left(p_{0}, q_{0}, q_{0}\right)\right) \leq q_{0}$. Since $p_{0} \not \leq q_{0}$, this is a contradiction. So we have proved the following

4.19. Theorem. The bi-frame $\mathbb{E}$ is not 3 -variable countably equationally compact. In fact, the system (4.8) is finitely solvable in $\mathbb{E}$ and not solvable in $\mathbb{E}$. 
Theorem 4.19 allows us immediately to go one step further. Recall that a Stone lattice [11, page 112] is a distributive lattice with 0 and 1 on which there exists a (necessarily unique) unary operation $x \mapsto x^{*}$ satisfying $(\forall x, y)\left(x \wedge y=0 \Leftrightarrow y \leq x^{*}\right)$ (i.e. $x \mapsto x^{*}$ is the pseudocomplementation on the lattice) and $(\forall x)\left(x^{*} \vee x^{* *}=1\right)$. For every bi-frame $A$, let $\varepsilon$ be an object not in $A$ and define on $A \cup\{\varepsilon\}$ a partial ordering extending the ordering of $A$ by $0<\varepsilon$ and $\varepsilon<x$ for all $x \in A \backslash\{0\}$. Denote by $A^{\varepsilon}$ the corresponding structure. It is easy to verify that $A^{\varepsilon}$ is also a bi-frame, but also a Stone lattice (of a very special kind, since $x^{*}=0$ for every $\left.x \neq 0\right)$. Note that $(A, \vee, \leq)$ is a substructure of $\left(A^{\varepsilon}, \vee, \leq\right)$ but that $(A, \wedge)$ may not be a substructure of $\left(A^{\varepsilon}, \wedge\right)$.

4.20. Corollary. Let $\mathbb{E}$ be the example of theorem 4.19. Then $\mathbb{E}^{\varepsilon}$ is a bi-frame and a Stone lattice but it is not 3-variable countably equationally compact.

Proof. Consider the following atomic system with unknowns $\mathbf{x}_{0}, \mathbf{x}_{1}$ and $\mathbf{x}_{2}$, with parameters from $\mathbb{E}^{\varepsilon}$ :

$$
\left\{\begin{array}{l}
A_{0}^{(m)} \leq \mathbf{x}_{0} \quad(\text { all } m \text { in } \omega) \\
A_{1}^{(0)} \leq \mathbf{x}_{1} \\
A_{2}^{(0)} \leq \mathbf{x}_{2} \\
\mathbf{x}_{0} \wedge \mathbf{x}_{1} \wedge \mathbf{x}_{2}=\varepsilon \\
\mathbf{x}_{0} \vee \mathbf{x}_{1} \vee \mathbf{x}_{2}=1
\end{array}\right.
$$

Since (4.8) is finitely solvable in $\mathbb{E},(4.12)$ is easily seen to be finitely solvable in $\mathbb{E}^{\varepsilon}$. But it is not solvable in $\mathbb{E}^{\varepsilon}$ : indeed, if $\left(x_{0}, x_{1}, x_{2}\right)$ is a solution triple of (4.12) in $\mathbb{E}^{\varepsilon}$, then the first three lines of (4.12) show that the $x_{i}$ belong to $\mathbb{E} \backslash\{0\}$, and thus the fourth line implies that $x_{0} \wedge x_{1} \wedge x_{2}=0$ in $\mathbb{E}$. Thus $\left(x_{0}, x_{1}, x_{2}\right)$ is a solution triple of $(4.8)$ in $\mathbb{E}$, a contradiction by theorem 4.19 .

The proof of corollary 4.20 shows actually that when it comes to equational compactness, bi-frames that are Stone lattices are about just as bad as bi-frames in general. It presents a strong contrast with an immediate consequence of [26, theorem 5.9] stating that if $A$ is a complete join-continuous Stone lattice satisfying the statement

$$
(\forall x, y)(\exists u, v)(x \leq y \vee u \text { and } y \leq x \vee v \text { and } u \wedge v=0)
$$

then $A$ is equationally compact (in particular, it is a bi-frame).

Note also that one step further, corollary 4.20 breaks down for Boolean algebras, since it is well-known that by Sikorski's extension theorem, complete Boolean algebras are equationally compact $[\mathbf{2 2}]$.

Note also finally that as for proposition 3.4, it is again possible to generalize the proof of theorem 4.19 to other partially ordered sets. For every partially ordered set $Q$, let $\mathcal{D}(Q)$ be the set of all sequences of the form $\left\langle X_{n}: n \in \omega\right\rangle$ where either all the $X_{n}$ 's are equal to a given nonempty finite subset of $Q$, or there exist $m \in \omega$ and $p, q, r$ in $Q$ such that for all $n<m, X(n)=\{p, q, r\}$ and for all $n \geq m, X(n)=\{q, r\}$. Thus if $Q$ is countable, then $\mathcal{D}(Q)$ is countable. Then define

$$
\Omega(Q)=\left\{(x, X) \in{ }^{\omega} Q \times \mathcal{D}(Q):(\forall n \in \omega)(x(n) \in X(n))\right\},
$$


endowed with the componentwise preordering defined as for $P$ after lemma 4.4. Then one can for example prove exactly the same way as previous theorem the following

4.21. Proposition. Let $Q$ be a [at most] countable partially ordered set with at least two elements, such that every increasing map $f: Q \times Q \rightarrow Q$ such that $(\forall q \in Q)(f(q, q) \leq q)$ is a projection. Then there are no residual subset $\Omega^{*}$ of $\Omega(Q)$ and no increasing map $f$ from $\Omega^{*}$ to $Q$ with the Baire property such that for all $(x, X)$ in $\Omega^{*}$ and all $q \in Q$ such that for all large enough $n$ in $\omega, X(n) \subseteq \downarrow\{q\}$, we have $f(x, X) \leq q$.

Note that we cannot a priori remove the assumption that $Q$ is countable: indeed, we need in several instances of the proof (as e.g. in lemma 4.10) to argue that intersections of families indexed by $Q$ of residual sets are residual.

4.22. Problem. When does there exist for a cardinal $\mathfrak{m}$ a bi-frame that is $\mathfrak{n}$-variable equationally compact for all $\mathfrak{n}<\mathfrak{m}$ but that is not $\mathfrak{m}$-variable equationally compact? (for $\mathfrak{m}$ at most countable, the bi-frame of all regular open lower subsets of some well-chosen $\Omega(Q)$ as above may be a solution; in the finite case, the natural guess would be to try the finite truncated Boolean lattices [7]).

4.23. Problem. Find the maximal amount of equational compactness satisfied by bi-frames (or $\sigma$-bi-frames) generated by Borel lower subsets of various preordered Polish spaces, for example for the $\sigma$-bi-frame of Borel lower subsets of $\mathbb{R}^{d}$ (with the natural ordering) where $d \leq \omega$.

\section{BIBLIOGRAPHY.}

[1] B. BANASCHEWSKI and E. NELSON, "Equational compactness in equational classes of algebras", Algebra Universalis 2 (1972), pp. 152-165.

[2] J.L. BELL, "On the strength of the Sikorski extension theorem for Boolean algebras", Journal of Symbolic Logic 48, $\mathrm{n}^{0} 3$ (1983), pp. 841-846.

[3] G. BIRKHOFF, "Lattice theory", American Mathematical Society Colloquium Publications, vol. 25, 3rd edition, seventh printing, 1993.

[4] S. BULMAN-FLEMING, I. FLEISCHER and K. KEIMEL, "The semilattices with distinguished endomorphisms which are equationally compact", Proceedings of the American Mathematical Society 73 (1979), $\mathrm{n}^{0} 1$, pp. 7-10.

[5] C.C. CHANG and H.J. KEISLER, "Model Theory", North Holland Publishing Company, 1973.

[6] J.P.R. CHRISTENSEN, "Topology and Borel structure", North Holland Publishing Company, 1974.

[7] E. COROMINAS, "Sur les ensembles ordonnés projectifs et la propriété du point fixe", Comptes Rendus de l'Académie des Sciences Paris, 311-I (1990), pp. 199-204.

[8] O. FRINK, "Topology in lattices", Transactions of the American Mathematical Society 51 (1942), pp. 569-582.

[9] S. GĂINĂ, "Order topologies in Boolean algebras", Revue Roumaine de Mathématiques Pures et Appliquées 17, $\mathrm{n}^{0} 2$ (1972), pp. 243-251. 
[10] G. GIERZ, K.H. HOFMANN, K. KEIMEL, J.D. LAWSON, M. MISLOVE and D.S. SCOTT, "A compendium of continuous lattices", Springer-Verlag, 1980.

[11] G. GRÄTZER, "General Lattice Theory", Birkhäuser Verlag, Basel, 1978.

[12] G. GRÄTZER and H. LAKSER, "Equationally compact semilattices", Colloquium Mathematicum 20, $\mathrm{n}^{0} 1$ (1969), pp. 27-30.

[13] D. K. HALEY, "Equational compactness in rings", Lecture Notes in Mathematics 745, Springer-Verlag, Berlin, Heidelberg, New-York, 1979.

[14] P.R. HALMOS, "Measure Theory", D. Van Nostrand, 1958.

[15] P.R. HALMOS, "Algebraic Logic", Chelsea Publishing Company, New York, 1962.

[16] P. JOHNSTONE, "Stone spaces", Cambridge University Press, Cambridge Studies in Advanced Mathematics 3, 1982.

[17] D. KELLY, "A note on equationally compact lattices", Algebra Universalis 2, $\mathrm{n}^{0} 1$ (1972), pp. 80-84.

[18] S. KOPPELBERG, "General theory of Boolean Algebras", in "Handbook of Boolean Algebras", vol. 1, pp. 1-307, edited by J.D. Monk with R. Bonnet, Elsevier, Amsterdam, 1989.

[19] K.A. NAURYZBAEV, "Equationally compact distributive lattices", Algebra and Logic 25 (1986), $\mathrm{n}^{0} 5$, pp. 369-379; translated from Algebra $i$ Logica, vol. 25, $\mathrm{n}^{0} 5$ (SeptemberOctober 1986), pp. 584-599.

[20] J. OXTOBY, "Measure and category", Graduate Texts in Mathematics 2, SpringerVerlag, New-York, Heidelberg, Berlin, 1971.

[21] P.S. REMA, "On compact topological lattices", Mathematica Japonica 9 (1964), n²2, pp. 93-98.

[22] R. SIKORSKI, "A theorem on extensions of homomorphisms", Ann. Soc. Polon. Math. 21 (1948), pp. 332-335.

[23] W. TAYLOR, "Some constructions of compact algebras", Annals of Mathematical Logic, vol. 3, n ${ }^{0} 4$ (1971), pp. 395-437.

[24] B. WȨGLORZ, "Equationally compact algebras (I)", Fundamenta Mathematica 59 (1966), pp. 289-298.

[25] B. WȨGLORZ, "Completeness and compactness of lattices", Colloquium Mathematicum 16 (1967), pp. 243-248.

[26] F. WEHRUNG, "Boolean universes above Boolean models", Journal of Symbolic Logic, vol. 58, n04 (december 1993), pp. 1219-1250.

[27] F. WEHRUNG, "A compactness property of Dedekind $\sigma$-complete $f$-rings", to appear in Algebra Universalis.

[28] F. WEHRUNG, "Bounded atomic compactness of ordered groups", to appear in Fundamenta Mathematica. 